\title{
Attributable Burden and Expenditure of Cardiovascular Diseases and Associated Risk Factors in Mexico and other Selected Mega-Countries
}

\author{
Kenny Mendoza-Herrera ${ }^{1}$, Andrea Pedroza-Tobías ${ }^{2}$, César Hernández-Alcaraz ${ }^{1}$,

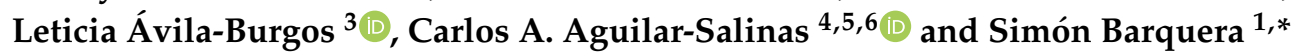 \\ 1 Center for Nutrition and Health Research, National Institute of Public Health, Cuernavaca, Morelos 62100, \\ Mexico; cinys26@insp.mx (K.M.-H.); cesar.hernandez@insp.mx (C.H.-A.) \\ 2 Institute for Global Health Sciences, University of California, San Francisco, CA 94158, USA; \\ Andrea.Pedroza-Tobias@ucsf.edu \\ 3 Center for Health Systems Research, National Institute of Public Health, Cuernavaca, Morelos 62100, Mexico; \\ leticia.avila@insp.mx \\ 4 Unidad de Investigación de Enfermedades Metabolicas, Mexico City 14080, Mexico; \\ caguilarsalinas@yahoo.com \\ 5 Departamento de Endocrinología y Metabolismo, Instituto Nacional de Ciencias Médicas y Nutrición \\ Salvador Zubiran, Mexico City 14080, Mexico \\ 6 Tecnologico de Monterrey, Escuela de Medicina y Ciencias de la Salud, Monterrey 64710, N.L., Mexico \\ * Correspondence: sbarquera@insp.mx; Tel.: +52-777-329-3017
}

Received: 20 August 2019; Accepted: 17 October 2019; Published: 22 October 2019

\begin{abstract}
Background: This paper describes the health and economic burden of cardiovascular diseases (CVD) in Mexico and other mega-countries through a review of literature and datasets. Methods: Mega-countries with a low (Nigeria), middle (India), high (China/Brazil/Mexico), and very high (the U.S.A./Japan) human development index were included. The review was focused on prevalence of dyslipidemias and CVD economic impact and conducted according to the PRISMA statement. Public datasets of CVD indicators were explored. Results: Heterogeneity in economic data and limited information on dyslipidemias were found. Hypertriglyceridemia and hypercholesterolemia were higher in Mexico compared with other countries. Higher contribution of dietary risk factors for cardiovascular mortality and greater probability of dying prematurely from CVD were observed in developing countries. From 1990-2016, a greater decrease in cardiovascular mortality in developed countries was registered. In 2015, a CVD expense equivalent to $4 \%$ of total health expenditure was reported in Mexico. CVD ranked first in health expenditures in almost all these nations and the economic burden will remain significant for decades to come. Conclusions: Resources should be assured to optimize CVD risk monitoring. Educational and medical models must be improved to enhance CVD diagnosis and the prescription and adherence to treatments. Long-term benefits could be attained by modifying the food system.
\end{abstract}

Keywords: Mexico; mega-countries; cardiovascular diseases; burden; expenditure

\section{Introduction}

Currently, cardiovascular diseases (CVD) are considered a major global health problem [1]. The global cumulative economic loss from not implementing strategies to tackle CVD and other chronic diseases from 2010 to 2030 is expected to be $\$ 47$ trillion US dollars (USD), which is equivalent to $75 \%$ of the 2010 gross domestic product (GDP) [2-5]. Due to the health and economic burden that these diseases generate, the World Health Organization (WHO) launched an action plan for prevention and 
control of chronic diseases for 2013-2020, creating a global initiative with a series of strategies to reduce the negative effects of CVD [3].

The high prevalence of obesity and non-communicable diseases (NCDs), as well as the increased mortality from these causes since the nineties, have placed Mexico in the spotlight of global health [6-8]. National health surveys in Mexico have identified dyslipidemias as the second most prevalent modifiable cardiovascular risk factor in adults, just after a body mass index (BMI) above $25 \mathrm{~kg} / \mathrm{m}^{2}$ [9]. As in other countries, a high low-density lipoprotein cholesterol (LDL-c) level has been described as the second most common risk factor for cardiovascular mortality in Mexico [10]. Since dyslipidemias are major contributors to the development of atherosclerosis [11], one of the main pathophysiological paths of CVD, their prevention and treatment are essential to counteract CVD burden and related expenditures. In order to direct strategies and allocate human and monetary resources to diminish the burden of lipid alterations, surveillance systems and studies that show the epidemiological status related to this health issue are needed.

Environmental factors have a crucial influence on the economic and health burden of CVD. As in other countries [12,13], the Mexican food system has undergone several transformations in recent decades. These modifications have caused a deterioration in diet, characterized by an increase in the consumption of ultra-processed food, sugar-sweetened beverages, and a low intake of fruits, vegetables, and whole grains [14-19]. This scenario has been associated with the increased prevalence of obesity, dyslipidemias, diabetes, and CVD, which represent important challenges for the health system and economy $[3,8,9,12-14,20]$.

In Mexico, CVD represent the leading cause of mortality, accounting for $\approx 20 \%$ of total deaths, from which $68.5 \%$ are caused by ischemic heart disease (IHD). Estimates indicate that $4 \%$ of total health expenditure in 2006 was spent on addressing CVD. Furthermore, investment in prevention strategies is much lower than in treatment and control actions [2]. Seventy-three percent of diabetes, obesity, and CVD expenditures are absorbed by three Mexican public health institutions: the Ministry of Health, the Mexican Social Security Institute (Spanish acronym: IMSS), and the Institute for Social Security and Services for State Workers (Spanish acronym: ISSSTE).

Sixty-five percent of global CVD mortality is concentrated in Mexico and the other 13 most populated mega-countries in the world. Among these countries, a great cultural diversity, distinct economic potential, and different epidemiological and nutrition transition stages can be observed. For example, although risk factors for NCDs, such as overweight and obesity, are not as much prevalent in Nigeria and India as in other mega-countries, even a low national prevalence of these conditions represents an enormous absolute number of affected people since they are two of the most populous countries in the world. Brazil, China, and Mexico, which are mainly dominated by a high prevalence of obesity and other risk factors, currently have economic systems that discourage healthy food systems and accelerate urbanization, thereby producing substantial changes in lifestyles in the last decades. The U.S.A. and Japan, two of the most developed mega-countries, are also experiencing a significant prevalence of NCDs. However, these two countries have been successful in decreasing the burden of some cardiovascular risk factors, and they have significantly more economic resources to address public health issues than other nations from this group [12].

Despite these differences, these countries have common political and economic characteristics that influence how the food and health system are regulated, which inevitably feed the global NCDs epidemic [12,21,22]. Because of this, understanding the dynamics of CVD-related epidemiological and economic scenarios in Mexico can contribute to identify effective strategies and critical financial measures to diminish the burden associated with these conditions. In addition, exploring and comparing this situation among other mega-countries can also be useful for visualizing reproducible solutions in this group of nations. Thus, this paper aims to describe the burden and national economic impact of CVD in Mexico, as well as to complementary compare these indicators from other selected mega-countries through a review of published literature and the exploration of secondary datasets. 


\section{Materials and Methods}

A literature review and exploration of secondary datasets with epidemiological indicators of CVD and their risk factors were carried out, in addition to a review on the expenditure generated by its medical costs in Mexico. Data from six selected mega-countries with a population of $\geq 100$ million [23] and a low (Nigeria), middle (India), high (China, Brazil, and Mexico), or very high (the U.S.A. and Japan) human development index (HDI) were also included. The HDI is composed of three indicators: (1) Health that considers life expectancy at birth; (2) education that considers the adult literacy rate and years of education; and (3) income with GDP per capita in international dollars. Thus, HDI allows for a comprehensive comparison between countries [24].

\subsection{Literature Review}

A literature review was conducted and reported following the standards provided by the PRISMA statement $[25,26]$ (PRISMA checklist (see Supplementary file 1)). Searches were first carried out in PubMed. Afterwards, to complement the review, a second search was performed considering the following order: ScienceDirect, Google Scholar, ResearchGate, and reference lists from studies. Duplicated studies found in the different stages were excluded from the final count. The review was conducted on two main topics: (1) Prevalence of dyslipidemias and (2) attributable economic burden to CVD, both nationwide. This review was carried out between January 2017 and December 2018.

The search period was limited to 2006 due to the availability of information on CVD costs in Mexico. As a result, to provide a standardized description of both topics, the first general inclusion criterion for the identification process was literature published in and related to the period between 2006 and 2018. The same search period was applied for the rest of the countries.

Identification, screening, filtering, and eligibility processes were performed for each topic. Searches were also limited to the availability of full abstracts and studies in humans. Search strategies were composed mainly of MeSH terms, but free vocabulary was also considered. Language restriction filters were not applied in the identification and screening processes. Eligibility and inclusion of studies were restricted to either English or Spanish, which are languages with which authors are familiar. Studies without clearly reported methods were excluded. Search strings are available in Supplementary Table S1 (see Supplementary file 2).

Given the implications of dyslipidemias for cardiovascular risk and the importance of actions against these lipid alterations to reduce CVD burden and expenditures described above, the first search was focused on the prevalence of high triglycerides (TG), high total cholesterol (TC), low high-density lipoprotein cholesterol (HDL-c), and high LDL-c in healthy adults $\geq 18$ years old. The terms used in this review were "dyslipidemia" (or "dyslipidemias") and "prevalence" (or "epidemiology"). Additionally, titles and abstracts of the identified articles were screened and filtered looking for the terms of lipid alterations, lipid abnormalities, proportion, patterns, trends, and population-based survey. For the eligibility process, the order of the following inclusion criteria and study characteristics were considered: (1) The most recent population-based surveys nationwide; (2) the most recent metanalyses or systematic reviews nationwide; (3) the most recent population-based surveys at regional level, and (4) studies with samples from multiple cities. Studies and information related to comorbidity of dyslipidemias with other conditions were excluded.

The second search was concentrated on estimates associated with economic burden attributable to CVD. CVD included in the 10th revision of the International Statistical Classification of Diseases and Related Health Problems (ICD-10) and their risk factors were considered in this stage. The terms used on this search were "cardiovascular disease" (or "cardiovascular diseases") and "cost" (or "economics"). The following vocabulary was considered for screening and filtering of abstracts and titles: expenditure, expenses, charges, spending, spent, economy, economic burden, economic impact, coronary heart disease (CHD), angina, heart disease, ischemic heart disease, myocardial infarction, myocardial ischemia, stroke, cerebral ischemic vascular event, ischemic vascular event, and vascular event. The main outcomes for this review were expenditures, rank of economic impact among different 
causes, fraction of relevant economic indicators spent on CVD, and projected economic losses and expenses. Full texts of cost-effectiveness studies were not reviewed. The following inclusion criteria were considered in the eligibility process: (1) Nationwide information; (2) report of monetary indicators, and (3) use of cost components, cost of illness methods, and modelling approaches. Microeconomic data were excluded (e.g., cost of treatment per patient year).

Identification and screening processes were carried out by one trained researcher. Afterwards, each potential article was fully reviewed in order to fill out the inclusion criteria checklist and to exclude studies that did not meet the criteria. All the studies classified as eligible were read by the research team and their inclusion decided by consensus. Data on authors, titles, databases, study design, year of estimation, main indicators, reported methodology, and main outcomes of the reviewed and included studies were extracted and summarized.

In order to standardize economic information, estimate expenditures from Mexico were deflated by considering the accumulated inflation until the year of the most recent information: 2006-2016= $46.81 \% ; 2008-2016=32.82 \% ; 2014-2016=5.56 \%$, and $2015-2016=3.36 \%$. Additionally, these estimates were adjusted by purchasing power parities (PPP) for 2016 (1 USD-PPP $=\$ 8.87$ pesos). Meanwhile, Mexican economic projections are presented as they appear in the literature. Economic data in currencies from other mega-countries were calculated to USD using the average yearly exchange rate. In addition, data from the studies that provide estimates in USD were kept and presented in this way.

\subsection{Review and Analysis of Secondary Databases}

Databases from the Mexican national surveys of health and nutrition (Spanish acronym: ENSANUT) 2006 and $2012[27,28]$ were analyzed to estimate the prevalence of obesity and hypertension in the northern, central-western, central, and southern regions of Mexico. Datasets of medicines purchases from the IMSS were reviewed in order to obtain information of expenditure on statins in Mexico [29].

Data from the Global Burden of Disease (GBD) study 2017 [10] related to age-standardized mortality rates of CVD and the attributable percentage of metabolic and dietary risk factors to cardiovascular deaths were reviewed. The metabolic risk factors were high systolic blood pressure, high LDL-c, high fasting plasma glucose, excess body weight, and impaired kidney function. The dietary risk factors were a low intake of nuts/seeds, vegetables, fruits, legumes, whole grains, fiber, polyunsaturated fatty acids (PUFAs) $\Omega-3$ from fish and seafood, a high intake of trans fatty acids, sodium, processed meat, and sugar-sweetened beverages. In the case of Mexico, information on IHD, ischemic stroke, CVD in general, and all the previous risk factors were considered. For the other selected countries, data on only CVD in general and four risk factors were included: (1) Low intake of seeds and grains; (2) low intake of polyunsaturated fatty acids (PUFAs) $\Omega-3$; (2) hypertension; and (3) hypercholesterolemia.

The WHO Global Health Observatory databases [30] were explored. From these, the indicator that reflects the probability of dying between 30 and 70 years old (premature death) from any CVD, cancer, diabetes, or chronic respiratory disease, was obtained. The information was reviewed for 2000 and 2016 in the seven mega-countries.

A narrative synthesis of the information identified in the literature reviews and the secondary datasets was conducted. In order to complement this article, some strategies to decrease the burden of CVD were discussed (no extensive review was performed for this particular literature).

\section{Results}

\subsection{Literature Search}

The detailed PRISMA flow diagram for the reviews on prevalence of dyslipidemias and CVD economic burden in seven mega-countries can be visualized in Figure 1. After removing duplicates, a total of 5776 studies were found on prevalence of dyslipidemias in the analyzed mega-countries. One hundred thirteen articles were fully reviewed and 13 of them were included. The number 
of identified studies on the economic burden of CVD in these nations was 8745 after duplicates, from which 201 were fully read and 19 included in this review. A summary of all the fully reviewed and included studies can be found in the Supplementary Tables S2-S5 (see Supplementary file 2).

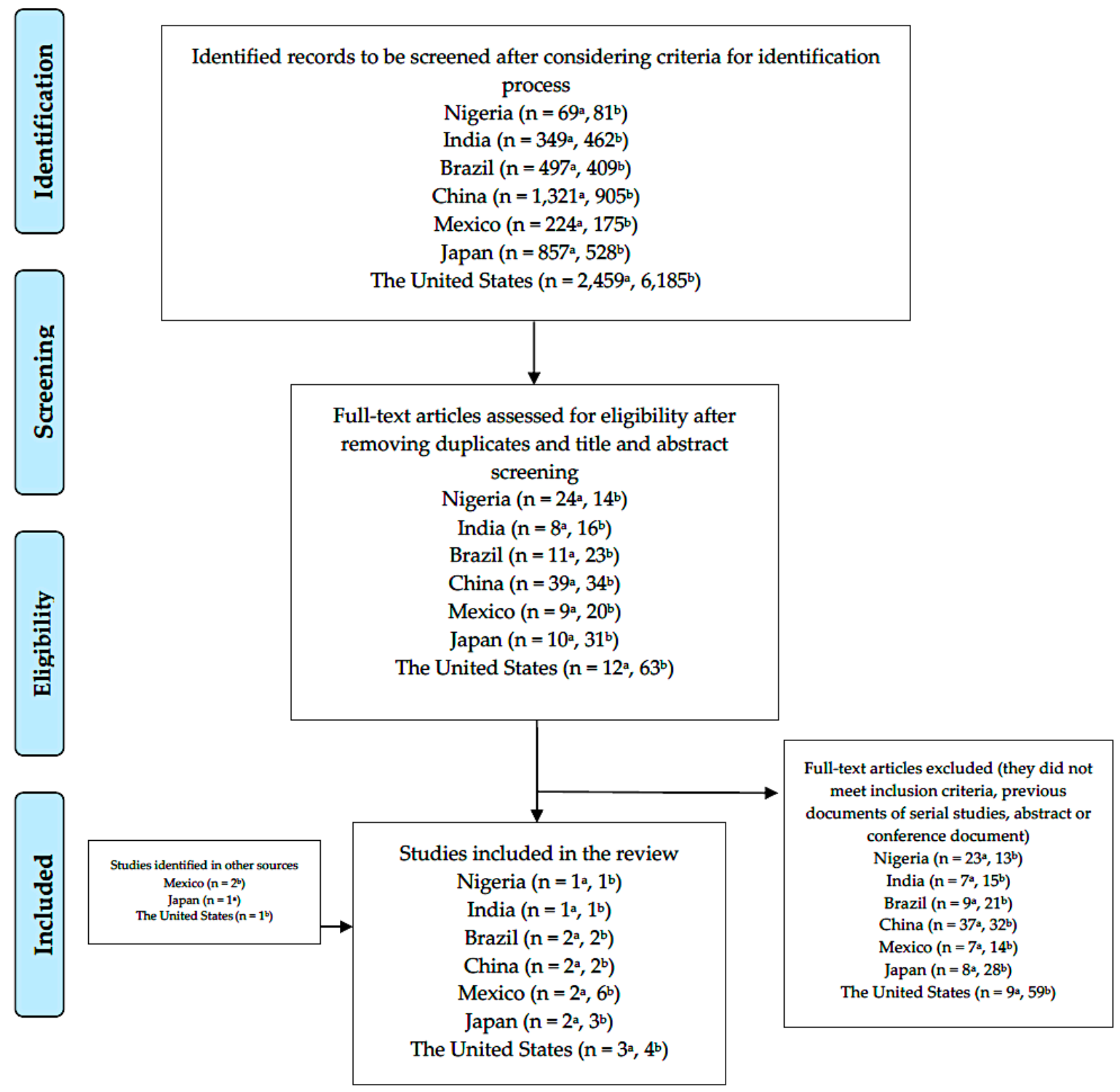

Figure 1. PRISMA flow diagram, literature review for seven mega-countries. a Search for dyslipidemias, b search for economic burden of cardiovascular diseases.

The nationwide and regional representative prevalence of hypertriglyceridemia, hypercholesterolemia, hypoalphalipoproteinemia, and high LDL-c, as well as the prevalence of Mexican adults eligible for pharmacological and lifestyle lipid-lowering therapy according to cardiovascular risk profiles in 2006, were obtained from two studies derived from ENSANUT [9,31]. Eligibility profiles in the latter study were based on lipid-lowering treatment recommendations considering the absolute risk of having CHD according to the National Cholesterol Education Program (NCEP) and the sex- and age-specific Framingham score tables [31,32].

The most recent information about the prevalence of dyslipidemias in the U.S.A. [33-35] and Japan [36,37] was obtained from studies based on analyses of the National Health and Nutrition Examination Survey (NHANES) and from a review on the Japanese National Health and Nutrition Survey 2015. For India, evidence of 2008-2010 from the Indian Council of Medical Research India 
Diabetes Study (ICMR-INDIAB study) was included [38]. Data from Nigeria were obtained from a study based on population-based surveys of four geopolitical areas [39], and data from China came from a 2014 metanalysis [40] and a nationally representative survey carried out between 2013 and 2014 [41]. In the case of Brazil, this information was collected from a prospective study with 15,105 participants [42] and the prevalence of self-reported high cholesterol levels was identified in a study based on the National Health Survey 2013 [43].

Expenditure estimates of CVD in Mexico were identified in a report from 2006, which included information on curative medical care, medical products, prevention actions, health management, laboratory services, research, and technological development in the Ministry of Health, IMSS, ISSSTE, and the private sector [2]. Estimates from the Ministry of Health and IMSS related to expenses on medical consultation, laboratory tests, medications, and hospitalization from addressing hypertension and IHD nationwide were obtained from a 2012 ENSANUT study [44].

Information on the expenditure generated by five CVD in Mexico in 2015 was identified through a study in press. These estimates considered the economic burden of medical treatment, and formal and informal medical care in the Mexican health system. In addition, they included the loss of productivity and welfare caused by hypertension, acute myocardial infarction, atrial fibrillation, and heart failure nationwide [45].

Expected total costs associated with hypertension for 2016 in Mexico, including indirect and direct expenditures for the Ministry of Health, IMSS, ISSSTE, and patients and families were identified in a study published in 2015 [46]. These estimates were based on national registries and were calculated through a modelling approach considering the changes in cases and demand of services in the health system. Another study from 2017 with similar sources and methodology was included. In this article, estimates concerned only older adults in Mexico [47]. Additionally, information on IMSS expenditures on atorvastatin and pravastatin in 2016 was obtained from the institution's online datasets of medicine purchases. These statins are part of the official catalogue of drugs from the Mexican public health sector.

Projections of the attributable expenditure to IHD, acute myocardial infarction, and stroke in Mexico were identified in a 2014 study. These estimates were based on USD from 2010 and three hypothetic scenarios of obesity rate changes between 2010 and 2050: (1) No reduction of the average BMI in the Mexican population; (2) reduction of 1.0\%, and (3) of 5.0\% [48-51]. These trends were based on BMI data from Mexican health and nutrition surveys of 1993, 1999, 2000, and 2006.

Data on the national economic burden of CVD in the other mega-countries were collected from a series of studies and reports [52-71]. Beginning with countries of a low and middle HDI, data of projected economic losses due to CVD in India between 2012 and 2030 were identified in a study based on the World Health Organization's EPIC model of economic growth. Information from Nigeria was obtained from a study that aimed to estimate the expected loss of GDP to 2015 in 23 low- and middle-income countries through an economic growth model.

Two studies related to CVD expenditures in China were included. The first one was related to estimates of foregone GDP projected to 2030 based on a human capital augmented production function, and the second one to hospitalization expenses in 2014. The included studies for Brazil concerned the economic impact of hypertension, heart failure, myocardial infarction, and atrial fibrillation including direct and indirect costs calculated through a cost of illness framework, as well as estimates on direct and indirect cost of CVD nationwide in 2015.

Evidence of CVD economic impact for mega-countries with a very high HDI were identified in seven sources. Expenditure estimates on ambulatory, emergency, inpatient, and nursing facility care, as well as pharmaceuticals in 2013 for the U.S.A were identified in one study. Data of direct and indirect costs between 2013 and 2035 were found in two reports from the American Heart Association. Current and projected estimates associated with informal caregiving for patients with CVD were obtained from another article (2015-2030, zero-inflated binomial model). For Japan, information on expenditures generated by direct medical care, morbidity and mortality, as well as by expenditures from insurance benefits and those incurred by family and friends for medical care at the household or 
community level, was identified in a single study. Other study based on the Cost of Illness method and modelling estimations reporting the economic impact of IHD in 2014, and between 2017 and 2029, was also included. In addition, projections of foregone GDP to 2030 based on a human capital augmented production function were identified.

A total of 16 reports and studies about strategies against CVD were reviewed for discussion. The included interventions were those identified by the WHO as the most cost-effective [3] and other similar strategies studied in isolation among the selected countries.

\subsection{CVD Risk Factors}

After obesity, hypoalphalipoproteinemia (HDL-c $<50 \mathrm{mg} / \mathrm{dL}$ for women and $<40 \mathrm{mg} / \mathrm{dL}$ for men) and high LDL-c $(\geq 100 \mathrm{mg} / \mathrm{dL})$ were the most prevalent cardiovascular risk factors in Mexican adults in 2006 (Table 1). In the same year, the prevalence of hypercholesterolemia in northern and central Mexico was higher than in the other regions.

Prevalence of hypertension (systolic/diastolic blood pressure $\geq 140 / \geq 90 \mathrm{~mm} \mathrm{Hg}$ ) in Mexico did not show substantial changes in the analyzed period. This condition was observed in three out of every ten Mexican adults and its prevalence in the northern region was the highest in 2006 and 2012. According to the newest data from the ENSANUT, prevalence of high blood pressure in Mexican adults was $25.5 \%$ in 2016 (data is not shown on tables) [72].

The most recent prevalence of hypertriglyceridemia and hypercholesterolemia in Mexico was higher compared with that in India, Nigeria (TG $\geq 150 \mathrm{mg} / \mathrm{dL}$ and TC $\geq 200 \mathrm{mg} / \mathrm{dL}$ ), China, Japan (TG $\geq 200 \mathrm{mg} / \mathrm{dL}$ and TC $\geq 240 \mathrm{mg} / \mathrm{dL}$ ), and in the U.S.A. (TG $\geq 150 \mathrm{mg} / \mathrm{dL}$ and TC $\geq 240 \mathrm{mg} / \mathrm{dL}$ ) (Tables 1 and 2). The highest prevalence of high LDL-c were found in Brazil (57.6\% (women) 58.5\% (men), LDL-c $\geq 130 \mathrm{mg} / \mathrm{dL}$ ), Mexico (46.0\%, LDL-c $\geq 130 \mathrm{mg} / \mathrm{dL}$ ), and in the U.S.A. (27\%, LDL-c $\geq 160 \mathrm{mg} / \mathrm{dL}$ (low risk groups), $\geq 130 \mathrm{mg} / \mathrm{dL}$ [intermediate-risk groups], and $\geq 100 \mathrm{mg} / \mathrm{dL}$ (high risk groups)). India had the highest prevalence of hypoalphalipoproteinemia ( $<50 \mathrm{mg} / \mathrm{dL}$ for women and $<40 \mathrm{mg} / \mathrm{dL}$ for men).

\subsection{Prevalence of Eligible Mexican Adults for Lipid-Lowering Therapy}

In 2006, 36.3\% of Mexican adults were eligible for therapy of lifestyle changes and $24.2 \%$ for pharmacological treatment considering their cardiovascular risk profile (Table 3) [31]. From the Mexican adults with cardiovascular risk profile one, $70.5 \%$ were eligible to follow pharmacological and lifestyle treatment. From the adults with diabetes, $71.4 \%$ were candidates for both treatments. Around $39.0 \%$ of the adults with two or more cardiovascular risk factors were candidates for lifestyles changes and $23.9 \%$ for medicines. The prevalence of Mexican adults with cardiovascular risk profiles two, three, and four that were candidates for treatment of lifestyle changes was $30.7 \%, 55.3 \%$, and $80.5 \%$ respectively. Meanwhile, the prevalence of adults with these profiles that required drugs was of $10.9 \%, 55.3 \%$, and $80.5 \%$, respectively [31].

\subsection{CVD Mortality Attributable to Metabolic and Dietary Risk Factors}

According to the GBD study in 2017, dietary risks factors contributed $48.4 \%$ to total CVD deaths, and $65.7 \%$ and $23.2 \%$ to the mortality associated with IHD and ischemic stroke in Mexico, respectively (Table 4). The intake of trans fatty acids contributed $6.7 \%$ to total CVD deaths and $10.2 \%$ to IHD deaths in the Mexican population. The percentage of CVD and IHD deaths attributable to low PUFAs $\Omega-3$ intake was $8.1 \%$ and $13.0 \%$ respectively. The contribution of low intake of PUFAs $\Omega-3$ and nuts/seeds to CVD mortality was higher in less developed countries (11.3\% and $13.0 \%$ India; $8.1 \%$ and $13.8 \%$ Mexico) compared to the developed countries ( $0.078 \%$ and $8.9 \%$ Japan; $6.5 \%$ and $10.7 \%$ the U.S.A.) [10]. Tables 2 and 4. 
Table 1. Prevalence of cardiovascular diseases (CVD) risk factors according to Mexican National Surveys, 2006 and 2012.

\begin{tabular}{|c|c|c|c|c|c|c|c|c|c|c|}
\hline & \multicolumn{2}{|c|}{ México } & \multicolumn{2}{|c|}{ North } & \multicolumn{2}{|c|}{ Center } & \multicolumn{2}{|c|}{ Center-West } & \multicolumn{2}{|c|}{ South } \\
\hline & $2006^{b}$ & $2012^{c}$ & $2006^{b}$ & $2012^{c}$ & $2006^{b}$ & $2012^{c}$ & $2006^{b}$ & $2012^{c}$ & $2006^{b}$ & $2012^{c}$ \\
\hline \multicolumn{11}{|c|}{ Metabolic conditions associated to CVD risk, prevalence (\%) } \\
\hline Overweight and obesity defined by BMI ${ }^{\mathrm{a}}$ & 69.7 & 71.3 & 71.5 & 72.8 & 70.2 & 71.2 & 69.3 & 70.2 & 66.6 & 71.0 \\
\hline Abdominal obesity ${ }^{a}$ & 75.7 & 74.0 & 79.7 & 76.6 & 74.6 & 76.4 & 78.1 & 74.7 & 71.7 & 71.6 \\
\hline Hypertension ${ }^{a}$ & 31.6 & 31.5 & 34.2 & 36.5 & 30.1 & 29.8 & 33.5 & 32.3 & 30.0 & 28.0 \\
\hline Hypertriglyceridemia ${ }^{\text {a }}$ & 31.5 [9] & - & 29.2 & - & 42.0 & - & 28.2 & - & 22.4 & - \\
\hline Hypercholesterolemia ${ }^{\text {a }}$ & $43.6[9]$ & - & 46.3 & - & 52.1 & - & 42.1 & - & 29.8 & - \\
\hline Hypoalphalipoproteinemia ${ }^{\text {a }}$ & 60.5 [9] & - & 58.3 & - & 49.3 & - & 65.9 & - & 72.8 & - \\
\hline High LDL-c ${ }^{\text {a }}$ & $46.0[9]$ & - & - & - & - & - & - & - & - & - \\
\hline
\end{tabular}

a Overweight and obesity defined as body mass index (BMI) $\geq 25 \mathrm{~kg} / \mathrm{m}^{2}$ and abdominal obesity as a waist circumference $\geq 80 \mathrm{~cm}$ for women and $\geq 90 \mathrm{~cm}$ for men; hypertension defined as systolic/diastolic blood pressure $\geq 140 / \geq 90 \mathrm{~mm} \mathrm{Hg}$; hypertriglyceridemia defined as triglycerides $\geq 150 \mathrm{mg} / \mathrm{dL}$, hypercholesterolemia as total cholesterol $\geq 200 \mathrm{mg} / \mathrm{dL}$, hypoalphalipoproteinemia as high-density lipoprotein cholesterol $<50 \mathrm{mg} / \mathrm{dL}$ for women and $<40 \mathrm{mg} / \mathrm{dL}$ for men and high low-density lipoprotein cholesterol (LDL-c) concentration as low-density lipoprotein cholesterol $\geq 130 \mathrm{mg} / \mathrm{dL} .{ }^{\mathrm{b}}$ National Survey of Health and Nutrition 2006 (Spanish acronym: ENSANUT 2006). ${ }^{\mathrm{c}}$ National Survey of Health and Nutrition 2012 (Spanish acronym: ENSANUT 2012). 
Table 2. Epidemiological and economic scenario related to CVD in seven mega-countries.

\begin{tabular}{|c|c|c|c|c|c|c|c|}
\hline & \multicolumn{2}{|c|}{ Low-Medium HDI } & \multicolumn{3}{|c|}{ High HDI } & \multicolumn{2}{|c|}{ Very High HDI } \\
\hline & India & Nigeria & México & China & Brazil & Japan & USA \\
\hline Hypertriglyceridemia, $\%^{\text {a }}$ & $29.5[38]$ & 23.4 [39] & $31.5[9]$ & $13.8[41]-17.7[40]$ & $\begin{array}{c}23.2 \text { (women) } \\
40.7 \text { (men) [42] }\end{array}$ & $18.0[36,37]$ & $30.0[34]$ \\
\hline Hypercholesterolemia, $\%^{\text {a }}$ & $13.9[38]$ & 25.9 [39] & $43.6[9]$ & $6.9[41]-10.1[40]$ & 12.5 (self-report) [43] & $16.2[36,37]$ & $12.4[33]$ \\
\hline Hypoalphalipoproteinemia, $\%^{\text {a }}$ & $72.3[38]$ & $43.8[39]$ & $60.5[9]$ & $11.0[40]-20.4[41]$ & $\begin{array}{l}20.7 \text { (women) } \\
14.7 \text { (men) [42] }\end{array}$ & $12.7[36,37]$ & 18.4 [33] \\
\hline High LDL-c, $\%^{a}$ & 11.8 [38] & - & $46.0[9]$ & $8.1[41]-8.8[40]$ & $\begin{array}{c}57.6 \text { (women) } \\
58.5 \text { (men) [42] }\end{array}$ & $11.1[36,37]$ & 27.0 [35] \\
\hline \multicolumn{8}{|l|}{ Probability of dying of $\mathrm{CVD}, \%^{\mathrm{b}}$} \\
\hline 2000 & 26.6 & 25.5 & 16.8 & 21.5 & 24.4 & 11.4 & 18.0 \\
\hline 2016 & 23.3 & 22.5 & 15.7 & 17.0 & 16.6 & 8.4 & 14.6 \\
\hline \multicolumn{8}{|c|}{ Age-standardized CVD deaths per 100,000 inhabitants ${ }^{c}$} \\
\hline 1990 & 317.7 & 248.8 & 195.8 & 332.3 & 341.8 & 183.7 & 256.1 \\
\hline 2017 & 282.3 & 181.0 & 152.8 & 261.9 & 178.0 & 79.4 & 151.1 \\
\hline$\%$ Change & -11.1 & -27.3 & -22.0 & -21.2 & -47.9 & -56.8 & -41.0 \\
\hline \multicolumn{8}{|c|}{ CVD mortality attributable to risk factors in $2017, \%^{\mathrm{c}}$} \\
\hline Low intake of nuts and seeds & $13.0[8.2,18.4]$ & $7.3[4.2,10.9]$ & $13.8[8.8,19.4]$ & $8.7[5.5,12.2]$ & $10.8[6.9,15.0]$ & $8.9[5.6,12.6]$ & $10.7[6.4,15.4]$ \\
\hline $\begin{array}{l}\text { Low intake of polyunsaturated } \\
\text { fatty acids (PUFAs) }(\Omega-3)\end{array}$ & $11.3[5.3,18.3]$ & $7.8[3.5,12.6]$ & $8.1[3.6,14.0]$ & $6.3[2.9,10.5]$ & $5.1[2.1,9.0]$ & $0.078[0.006,0.27]$ & $6.5[2.7,11.7]$ \\
\hline High systolic blood pressure & $53.0[47.1,58.9]$ & $58.6[52.2,64.5]$ & $49.7[42.6,57.0]$ & $54.5[47.9,61.0]$ & $53.3[47.9,58.8]$ & $49.4[43.1,55.6]$ & $44.5[37.8,51.1]$ \\
\hline High LDL cholesterol & $24.1[18.4,30.2]$ & $15.9[11.0,22.0]$ & $30.0[22.5,38.1]$ & $19.6[14.2,26.2]$ & $25.1[20.0,30.6]$ & $22.1[16.2,29.2]$ & $25.1[18.8,32.1]$ \\
\hline \multicolumn{8}{|l|}{ Expenditure on CVD nationwide ${ }^{d}$} \\
\hline Total, \$USD & $\begin{array}{c}\text { Cumulative gross } \\
\text { domestic product } \\
\text { loss associated with } \\
\text { coronary heart } \\
\text { disease, stroke, and } \\
\text { diabetes (2006-2015) } \\
\$ 16.7 \text { billion [61] }\end{array}$ & $\begin{array}{c}\text { Cumulative gross } \\
\text { domestic product } \\
\text { loss associated with } \\
\text { coronary heart } \\
\text { disease, stroke, and } \\
\text { diabetes (2006-2015) } \\
\$ 1.17 \text { billion [61] }\end{array}$ & $\begin{array}{c}\text { Hypertension, myocardial } \\
\text { infarction, atrial fibrillation } \\
\text { and heart failure only [45]: } \\
2016 \text { USD- purchasing } \\
\text { power parities (PPP) } 11.3 \\
\text { billion (2015) }\end{array}$ & $\begin{array}{c}\text { Hospitalization } \\
\text { expenses related to } \\
\text { acute myocardial } \\
\text { infarction, intracranial } \\
\text { hemorrhage, } \\
\text { and cerebral infarction: } \\
\$ 13.2 \text { billion } \\
(2014)[57]\end{array}$ & $\begin{array}{l}\$ 11.1 \text { billion (2015) [60] } \\
\text { Economic impact of } \\
\text { hypertension, heart } \\
\text { failure, } \\
\text { myocardial infarction, } \\
\text { and atrial fibrillation } \\
\$ 17.4 \text { billion [68] (2015) }\end{array}$ & $\begin{array}{l}\$ 109.6 \text { billion (2014) [59] } \\
\text { Costs associated with } \\
\text { ischemic heart disease: } \\
\$ 16.13 \text { billion (2014) [71] }\end{array}$ & $\begin{array}{l}\text { \$316.1 billion (2013) [55] } \\
\text { CVD: } \\
\$ 555 \text { billion (2016) [69] } \\
\text { Costs of informal } \\
\text { caregiving for patients } \\
\text { with CVD: } \\
\$ 61 \text { billion (2015) [70] }\end{array}$ \\
\hline Rank nationwide ${ }^{e}$ & $\begin{array}{l}\text { First (among } \\
\text { projected economic } \\
\text { losses due to CVD, } \\
\text { diabetes, chronic } \\
\text { respiratory disease, } \\
\text { cancer, and mental } \\
\text { health conditions to } \\
\text { 2030) [66] }\end{array}$ & - & $\begin{array}{c}\text { First (among CVD, obesity } \\
\text { and diabetes } \\
\text { expenditures) [2] }\end{array}$ & $\begin{array}{c}\text { Second (among } \\
\text { projected expenditures } \\
\text { due to CVD, diabetes, } \\
\text { chronic respiratory } \\
\text { disease, cancer, and } \\
\text { mental health } \\
\text { conditions to } 2030 \text { [67] }\end{array}$ & - & $\begin{array}{c}\text { First (among } \\
\text { expenditures generated } \\
\text { by cancer, heart disease, } \\
\text { and cerebrovascular } \\
\text { disease) [59] }\end{array}$ & $\begin{array}{c}\text { First (among } \\
\text { expenditures generated } \\
\text { by } 14 \text { aggregated } \\
\text { condition } \\
\text { categories) [53] }\end{array}$ \\
\hline
\end{tabular}


Table 2. Cont.

\begin{tabular}{|c|c|c|c|c|c|c|c|}
\hline & \multicolumn{2}{|c|}{ Low-Medium HDI } & \multicolumn{3}{|c|}{ High HDI } & \multicolumn{2}{|c|}{ Very High HDI } \\
\hline & India & Nigeria & México & China & Brazil & Japan & USA \\
\hline $\begin{array}{l}\text { Fraction of different relevant } \\
\text { economic indicators spent on CVD } \\
\text { nationwide, } \%\end{array}$ & - & - & $\begin{array}{l}\text { Hypertension and ischemic } \\
\text { heart disease (IHD) only } \\
\text { (2012) [44]: } \\
2 \% \text { from the Ministry of } \\
\text { Health's total medical } \\
\text { expenditure } \\
\text { 8.1\% from I Mexican Social } \\
\text { Security Institute (Spanish } \\
\text { acronym: IMSS)' total } \\
\text { medical expenditure } \\
0.13 \% \text { from GDP } \\
\text { Hypertension, myocardial } \\
\text { infarction, atrial fibrillation } \\
\text { and heart failure (2015) [45]: } \\
4 \% \text { from total medical } \\
\text { expenditure }\end{array}$ & - & $\begin{array}{l}0.7 \% \text { from GDP } \\
\text { (2010-2015) [60] }\end{array}$ & - & $\begin{array}{l}11 \% \text { from total medical } \\
\text { expenditure (2013) [53] }\end{array}$ \\
\hline $\begin{array}{l}\text { Projected expenditure on CVD, } \\
\text { \$USD }^{\mathrm{d}}\end{array}$ & $\begin{array}{l}\text { Economic losses due } \\
\text { CVD: } \\
\$ 2.17 \text { trillion } \\
(2012-2030)[66]\end{array}$ & $\begin{array}{l}\text { Cumulative gross } \\
\text { domestic product } \\
\text { loss associated with } \\
\text { coronary heart } \\
\text { disease, stroke, and } \\
\text { diabetes: } \\
\$ 1.17 \text { billion } \\
(2006-2015) \text { [61] }\end{array}$ & $\begin{array}{l}\$ 9.3 \text { billion }(2010-2030) \\
\$ 23.5 \text { billion } \\
(2010-2050)[51]\end{array}$ & $\begin{array}{c}\text { Economic losses due } \\
\text { to CVD: } \\
\$ 1.5 \text { trillion } \\
(2010-2030)[67]\end{array}$ & - & $\begin{array}{c}\text { Economic losses due } \\
\text { CVD } \\
\$ 756 \text { billion } \\
(2010-2030)[67] \\
\text { Predicted cost } \\
\text { associated with ischemic } \\
\text { heart disease [71]: } \\
\$ 15.3 \text { billion (2017) } \\
\$ 11.5 \text { billion }(2029)\end{array}$ & $\begin{array}{l}\text { \$918 billion (2030) [55] } \\
\text { CVD: } \\
\$ 1.1 \text { trillion (2035) [69] } \\
\text { Costs of informal } \\
\text { caregiving for patients } \\
\text { with CVD: } \\
\$ 128 \text { billion (2035) [70] }\end{array}$ \\
\hline $\begin{array}{l}\text { Specific estimates of potential } \\
\text { savings (\$USD) or diminishable } \\
\text { burden of CVD due to } \\
\text { prevention and control policies }{ }^{d}\end{array}$ & $\begin{array}{l}20 \% \text { taxation on } \\
\text { palm oil purchases } \\
\text { in } 10 \text { y [64]: } \\
\approx 363,000 \text { deaths } \\
\text { from myocardial } \\
\text { infarctions (1.3\% } \\
\text { absolute reduction } \\
\text { in CVD deaths) } \\
\text { Reduction in salt } \\
\text { intake by } 3 \mathrm{~g} / \mathrm{d} \text { in } \\
30 \text { y [63]: } \\
\approx 400,000 / 81,000 \\
\text { CVD events/deaths }\end{array}$ & - & $\begin{array}{c}\text { Reduction on averaged IMC } \\
\text { (2010-50): } \\
<1 \% \$ 986 \text { million }<5 \% \$ 2.1 \\
\text { billion [51] } \\
\text { A } 10 \% \text { reduction in } \\
\text { consumption of } \\
\text { sweetened-sugar beverages } \\
\text { in } 10 \text { y [73]: } \\
46,300 / 9300 \text { coronary heart } \\
\text { disease cases/deaths } \\
6200 / 1600 \text { stroke } \\
\text { cases/deaths } \\
14,200 \text { myocardial } \\
\text { infarctions }\end{array}$ & $\begin{array}{c}\text { Extension and } \\
\text { optimization of } \\
\text { coverage of } \\
\text { lipid-lowering and } \\
\text { antihypertensive } \\
\text { treatments, } \\
\text { (2016-2030) [54]: } \\
\$ 932 \text { billions } \\
850,000 \text { cases of } \\
\text { myocardial infarction } \\
\text { 300,000 CVD deaths } \\
\text { Reducing CVD } \\
\text { mortality by } 1 \% \text { per } \\
\text { year }(2010-2040): \\
\$ 10.7 \text { trillion [58] }\end{array}$ & - & $\begin{array}{c}\text { Reduction of } \approx 66 \% \text { in } \\
\text { the risk of } \\
\text { cardiovascular events, } \\
\text { associated to quitting } \\
\text { smoking for four years } \\
\text { or more in healthy adult } \\
\text { men [74] }\end{array}$ & $\begin{array}{l}\text { Investment of } \$ 10 \\
\text { USD/person/year in } \\
\text { community programs to } \\
\text { address lifestyle } \\
\text { modifiable risk } \\
\text { factors [56]: } \\
\$ 18 \text { billion USD } \\
\text { Optimal compliance } \\
\text { with guidelines for the } \\
\text { management of } \\
\text { hypertension: } \\
\text { Annual reduction of } \\
\text { 56,000 events and 13,000 } \\
\text { CVD deaths [75] }\end{array}$ \\
\hline
\end{tabular}

${ }^{a}$ Data of dyslipidemias prevalence was obtained from population-based studies or reviews from each country. ${ }^{b}$ Probability (\%) of dying between age 30 and exact age 70 from any of cardiovascular disease, cancer, diabetes, or chronic respiratory disease (Global strategy for women's, children's and adolescents' health), indicator from Global Health Observatory, World

Health Organization. ' ${ }^{c}$ Data from the Institute for Health Metrics and Evaluation (IHME). Global Burden of Disease (GBD) Compare. Seattle, WA: IHME, University of Washington, 2017.

${ }^{\mathrm{d}}$ Estimates among countries can variate according to methodologies and designs from the information sources. ${ }^{\mathrm{e}}$ Rank among different causes included in the information sources. 
Table 3. Prevalence of Mexican adult candidates for treatments according to cardiovascular risk profiles, 2006.

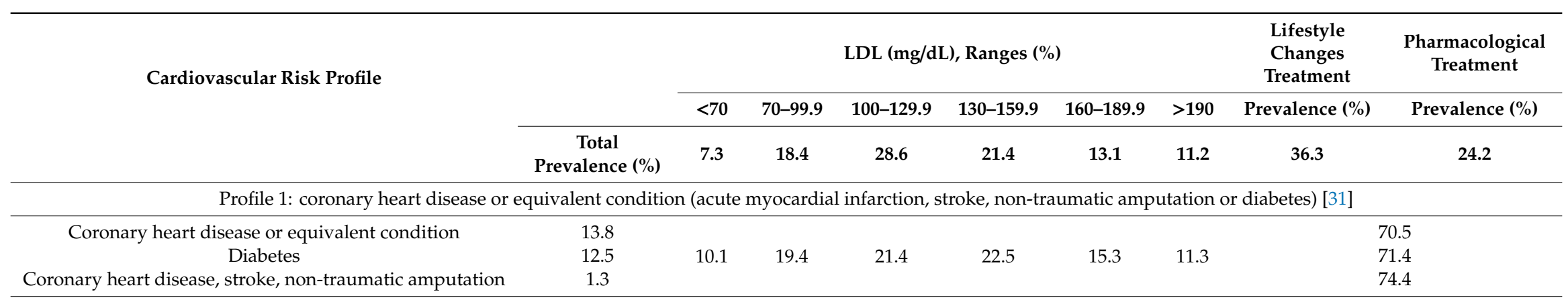

Without coronary heart disease or equivalent condition [31]

Two or more CVD risk factors (age $\geq 45$ years for men and $\geq 55$ years for women, family history of coronary disease, current smoking or hypertension)

\begin{tabular}{|c|c|c|c|c|c|c|c|c|c|}
\hline Profile 2: $\geq 2$ CVD risk factors + Framingham score $<10 \%$ & 23.3 & 9.6 & 24.9 & 34.7 & 19.8 & 7.0 & 3.9 & 30.7 & 10.9 \\
\hline Profile 3: $\geq 2$ CVD risk factors + Framingham score $10-20 \%$ & 5.9 & 4.0 & 18.4 & 22.2 & 21.3 & 18.8 & 15.2 & 55.3 & 55.3 \\
\hline Profile 4: $\geq 2$ CVD risk factors + Framingham score $>20 \%$ & 2.2 & 3.8 & 15.8 & 12.9 & 10.1 & 27.2 & 30.3 & 80.5 & 80.5 \\
\hline
\end{tabular}

Information adapted from: Gómez-Pérez F, Rojas R, Villalpando S, Barquera S, Rull J, Aguilar-Salinas C. Prevention of cardiovascular disease based on lipid lowering treatment: a challenge for the Mexican health system. Salud Publica De Mexico. 2010; 52 Suppl 1S54-S62 [31]. 
Table 4. Age-standardized deaths of CVDs attributable to risk factors in Mexico, 2017.

\begin{tabular}{|c|c|c|c|}
\hline CVD Risk Factors & $\begin{array}{c}\text { Cardiovascular Diseases }^{a}, \\
\text { Deaths per 100,000 Inhabitants } \\
\text { [Uncertainty Interval] (\%) }{ }^{b}\end{array}$ & $\begin{array}{c}\text { Ischemic Heart Disease, Deaths } \\
\text { per 100,000 Inhabitants } \\
\text { [Uncertainty Interval] (\%) }\end{array}$ & $\begin{array}{c}\text { Ischemic Stroke, Deaths per } \\
100,000 \text { Inhabitants } \\
\text { [Uncertainty Interval] (\%) }\end{array}$ \\
\hline \multicolumn{4}{|l|}{ Attributable Burden of Dietary Risk Factors } \\
\hline Total & $73.9[65.3,83.5](48.4)$ & $63.0[54.6,71.5](65.7)$ & $3.4[2.5,4.4](23.2)$ \\
\hline Low intake of nuts and seeds & $21.1[13.4,29.7](13.8)$ & $21.1[13.4,29.7](22.0)$ & . \\
\hline Low intake of vegetables & $17.5[8.6,28.3](11.5)$ & $14.4[5.7,24.6](15.1)$ & $1.0[0.3,1.8](6.7)$ \\
\hline Low intake of PUFAs $(\Omega-3)$ from fish and seafood & $12.4[5.5,21.2](8.1)$ & $12.4[5.5,21.2](13.0)$ & \\
\hline Low intake of fruits & $11.3[5.3,19.1](7.4)$ & $7.0[2.2,13.5](7.3)$ & $1.4[0.6,2.3](9.3)$ \\
\hline High intake of trans fatty acids & $10.2[6.0,15.9](6.7)$ & $10.2[6.0,15.9](10.7)$ & \\
\hline High intake of sodium & $7.2[0.1,22.4](4.7)$ & $4.6[0.1,14.3](4.8)$ & $0.6[0.01,1.9](3.9)$ \\
\hline Low intake of fiber & $4.2[1.8,7.6](2.7)$ & $4.2[1.8,7.6](4.3)$ & . \\
\hline Low intake of polyunsaturated fatty acids & $7.8[3.3,13.1](5.1)$ & $7.8[3.3,13.1](8.2)$ & . \\
\hline High intake of processed meat & $2.4[0.1,4.9](1.6)$ & $2.4[0.1,4.9](2.5)$ & . \\
\hline Low intake of legumes & $1.8[0.5,4.1](1.2)$ & $1.8[0.5,4.1](1.9)$ & . \\
\hline High intake of sugar-sweetened beverages & $8.6[-](5.6)$ & $8.6[-](8.9)$ & . \\
\hline Low intake of whole grains & $11.5[5.7,18.9](7.5)$ & $8.8[4.2,15.1](9.1)$ & $0.9[0.5,1.5](6.2)$ \\
\hline \multicolumn{4}{|l|}{ Attributable Burden of Metabolic Risk Factors } \\
\hline Total & $118.3[109.4,126.6](77.4)$ & $80.6[72.9,88](84.1)$ & $10.1[8.2,12.2](68.6)$ \\
\hline High systolic blood pressure & $75.9[65.0,87.0](49.7)$ & $46.6[36.4,56.8](48.6)$ & $6.2[4.5,8.0](41.9)$ \\
\hline High LDL cholesterol & $45.8[34.5,58.2](30.0)$ & $42.7[31.6,54.4](44.6)$ & $3.0[1.0,6.4](20.7)$ \\
\hline High fasting plasma glucose & $46.6[30.6,68.2](30.5)$ & $35.4[20.4,55.9](37.0)$ & $4.6[2.2,9.9](31.2)$ \\
\hline Overweight and obesity & $36.5[23.1,50.3](23.9)$ & $22.5[13.5,32.2](23.5)$ & $2.1[1.2,3.2](14.3)$ \\
\hline Impaired kidney function & $17.2[14.5,20.2](11.3)$ & $13.7[11.2,16.4](14.3)$ & $1.6[1.1,2.2](10.9)$ \\
\hline
\end{tabular}

${ }^{a}$ It includes all CVDs considered by Global Burden of Disease Study by 2017. ${ }^{b}$ It refers to the attributable percentage from risk factors to the total of CVD deaths. Information adapted from: Institute for Health Metrics and Evaluation (IHME). GBD Compare Data Visualization. Seattle, WA: IHME, University of Washington, 2017. Available from http://vizhub.healthdata.org/gbd-compare. Accessed 20 November 2018. 
Metabolic risk factors were associated with $77.4 \%$ of total CVD deaths, $84.1 \%$ of the IHD deaths, and $68.6 \%$ of the ischemic stroke mortality in Mexico. High LDL-c ranked second among the metabolic risk factors with major influence on mortality caused by CVD and IHD (just after high systolic blood pressure), with a contribution of $30.0 \%$ and $44.6 \%$ to the deaths from these conditions in Mexican individuals. This lipid abnormality ranked third among metabolic risk factors that contributed most to ischemic stroke mortality, with $20.7 \%$ of its deaths. In every single selected country, the contribution of the high LDL-c to total CVD deaths was equal to or higher than $15.9 \%$, and in Mexico it had the highest contribution [10]. Tables 2 and 4.

Probability of premature death by CVD and other chronic diseases in 2016 was of $23.3 \%-22.5 \%$ for low-middle HDI countries, 17.0-15.7\% for high HDI countries, and of $14.6 \%-8.4 \%$ for very high HDI countries [30]. From 1990 to 2017, a relative decrease in age-standardized CVD mortality rates of $11.1 \%, 27.3 \% 22.0 \%, 21.2 \%, 47.9 \%, 56.8 \%$, and $41.0 \%$ in India, Nigeria, Mexico, China, Brazil, Japan, and the U.S.A., was observed respectively [30] (Table 2).

\subsection{Attributable Expenditures to CVD}

In 2006, CVD care concentrated 55\% of the total public and private health expenditures directed to address diabetes, obesity, and CVD in Mexico. The expenditures on CVD in USD-PPP were $\$ 812.5$ million in the ISSSTE, $\$ 1.8$ billion in the IMSS, and $\$ 343.2$ million in the Ministry of Health. The equivalent percentages of the annual budget of these institutions were $23.2 \%, 11.0 \%$, and $2.4 \%$, respectively. Between $48.4 \%$ and $66.0 \%$ of the total expenditures on chronic diseases in these three institutions corresponded to CVD. The percentage of the annual budget spent on CVD drugs was $2.8 \%$ in the ISSSTE, $1.9 \%$ in the IMSS, and $0.2 \%$ in the Ministry of Health. The expenditures on control and prevention actions for CVD from the annual budget of the Ministry of Health was $2.3 \%$ and $0.02 \%$, respectively [2].

In 2012, the IMSS allocated USD-PPP $\$ 1.3$ billion and USD-PPP $\$ 719.8$ million to address hypertension and IHD respectively, including ambulatory consultation, laboratory tests, drugs, and hospitalization expenditures. The Ministry of Health spent USD-PPP \$425.9 million on hypertension and USD-PPP \$107 million on IHD in the same areas [44]. In 2015, hypertension, myocardial infarction, atrial fibrillation, and heart failure represented an expense of USD-PPP \$11.2 billion in Mexico, including the loss of productivity and welfare [45].

The Ministry of Health, IMSS, and ISSSTE were expected to respectively allocate USD-PPP \$1.6, $\$ 2.7$, and $\$ 1.1$ billion to address the increased demand of health services associated with hypertension in 2016. The expenditures expected for users surpassed the sum of these previous amounts, being USD-PPP $\$ 5.6$ billion [46]. A similar pattern in the proportion of these expenses among health care providers and users over 60 years old was observed by 2015 [47]. In 2016, the IMSS spent USD-PPP $\$ 6.6$ million on atorvastatin and USD-PPP $\$ 16.1$ million on pravastatin, and the average unit price of these statins was USD-PPP \$1.1 and USD-PPP \$1.08, respectively [29] (Table 5).

In the case that the average BMI in the Mexican population remains static from 2010 to 2050, the economic burden of IHD, myocardial infarction, and stroke would mean a nationwide accumulated expenditure of USD $\$ 23$ billion. A reduction of $1.0 \%$ in the average BMI in the population would reduce USD $\$ 986$ million from the accumulated CVD expenditures in this period, while a decrease of $5.0 \%$ would save USD $\$ 753$ million to 20 years and USD $\$ 2.1$ billion to 40 years [51] (Figure 2).

CVD ranked first among expenditures derived from health care for different causes in India, Mexico, Japan, and the U.S.A., and it represented the second place in China. The highest expenditure due to CVD was reported in the evidence from the U.S.A., USD \$316.1 billion in 2013 [55] and \$555 billion in 2016 [69]. Projections indicate that the economic losses generated by CVD will be of USD $\$ 918$ billion [55]-\$1.1 trillion [69], USD \$756 billion [67], USD \$2.17 trillion [66], and of \$1.5 trillion [67] for the U.S.A., Japan, India, and China by 2030-2035, respectively. One study in Japan indicated an expected decrease in the economic burden generate by IHD, reducing from USD \$15.3 billion in 2017 to USD $\$ 11.5$ billion by 2029 [71]. Projections were not found for Brazil (Table 2). 
Table 5. Estimated expenditures for CVD in Mexico expressed in USD-PPP and pesos a , 2006-2016.

\begin{tabular}{|c|c|c|c|c|c|}
\hline \multirow{2}{*}{ Author } & \multirow{2}{*}{ Year } & \multirow{2}{*}{ CVD/Item } & \multirow{2}{*}{ Institutions } & \multicolumn{2}{|c|}{ Total Expenditure Nationwide } \\
\hline & & & & USD-PPP & Pesos \\
\hline \multirow{3}{*}{ Ávila-Burgos et al. [2] } & \multirow{3}{*}{2006} & \multirow{3}{*}{$\begin{array}{l}\text { Ischemic heart disease, cerebrovascular diseases, } \\
\text { hypertension, peripheral vascular diseases, } \\
\text { rheumatic diseases and rheumatic heart diseases, } \\
\text { congestive heart failure, pulmonary heart } \\
\text { disease and other heart disease }\end{array}$} & $\begin{array}{l}\text { Ministry of Health } \\
\text { IMSS }\end{array}$ & $\begin{array}{l}343,152,856\left(48.4 \%^{b}\right)\left(2.4 \%^{c}\right) \\
1,766,349,589\left(61.3^{b}\right)\left(11.0 \%^{c}\right)\end{array}$ & $\begin{array}{c}3,043,422,684 \\
15,665,754,501\end{array}$ \\
\hline & & & Institute for Social Security and Services for & $812,492,790\left(66 . \%^{b}\right)\left(23.2^{c}\right)$ & $7,205,998,550$ \\
\hline & & & $\begin{array}{l}\text { State Workers (Spanish acronym: ISSSIE) } \\
\text { Private sector }\end{array}$ & $723,092,634(40.5 \%$ b $)$ & $6,413,108,571$ \\
\hline \multirow{6}{*}{ Figueroa-Lara et al. [44] } & \multirow{6}{*}{2012} & \multirow{3}{*}{ Hypertension } & Ministry of Health & $425,901,122$ & $3,777,317,057$ \\
\hline & & & IMSS & $1,312,998,401$ & $11,644,982,822$ \\
\hline & & & Ministry of Health & $107,039,658$ & $949,334,732$ \\
\hline & & Ischemic heart disease & IMSS & $719,802,391$ & $6,383,927,403$ \\
\hline & & \multirow{2}{*}{ Both } & Ministry of Health & $532,940,781\left(2 \%^{c}\right)$ & $4,726,651,789$ \\
\hline & & & IMSS & $2,032,800,792\left(8.1 \%^{c}\right)$ & $18,028,910,225$ \\
\hline \multirow{5}{*}{ Stevens B et al. [45] } & \multirow{5}{*}{2015} & Hypertension & \multirow{5}{*}{$\begin{array}{l}\text { Complete health system including loss of } \\
\text { productivity and welfare }\end{array}$} & $2,645,591,792$ & 23463753600 \\
\hline & & Heart failure & & $3,148,115,549$ & $27,920,636,800$ \\
\hline & & Myocardial infarction & & $4,550,683,595$ & $40,360,012,800$ \\
\hline & & Atrial fibrillation & & $973,931,131$ & $8,637,795,200$ \\
\hline & & Total & & $11,233,946,555\left(4 \%{ }^{d}\right)$ & $99,633,872,000$ \\
\hline \multirow{5}{*}{ Arredondo et al. [47] } & \multirow{5}{*}{2015} & \multirow{5}{*}{ Hypertension (adults over 60 years old) } & Ministry of Health & $585,138,637.9$ & $5,189,594,579$ \\
\hline & & & IMSS & $985,625,322.1$ & $8,741,510,981$ \\
\hline & & & ISSSTE & $394,118,027$ & $3,495,432,782$ \\
\hline & & & Users & $2,051,383,788$ & $18,193,722,816$ \\
\hline & & & Total & $4,022,328,467$ & $35,674,031,175$ \\
\hline \multirow{5}{*}{ Arredondo et al. [46] } & \multirow{5}{*}{2016} & \multirow{5}{*}{ Hypertension } & Ministry of Health & $1,604,264,783$ & $14,228,224,358$ \\
\hline & & & IMSS & $2,674,376,729$ & $23,719,047,208$ \\
\hline & & & ISSSTE & $1,069,487,777$ & $9,485,287,094$ \\
\hline & & & Users & $5,566,411,341$ & $49,368,502,187$ \\
\hline & & & Total & $10,914,540,614$ & $96,801,060,709$ \\
\hline \multirow{5}{*}{ IMSS datasets [29] } & \multirow{5}{*}{2016} & Total expenditure on statins by IMSS in 2016 & & & \\
\hline & & Atorvastatin & & $6,594,810$ & $58,489,369$ \\
\hline & & Pravastatin & Estimates from IMSS datasets & $16,076,150$ & $142,579,376$ \\
\hline & & $\begin{array}{l}\text { Average unit price in } 2016 \\
\text { Atorvastatin }\end{array}$ & & 11 & 99 \\
\hline & & Pravastatin & & $\begin{array}{l}1.1 \\
1.08\end{array}$ & 9.6 \\
\hline
\end{tabular}




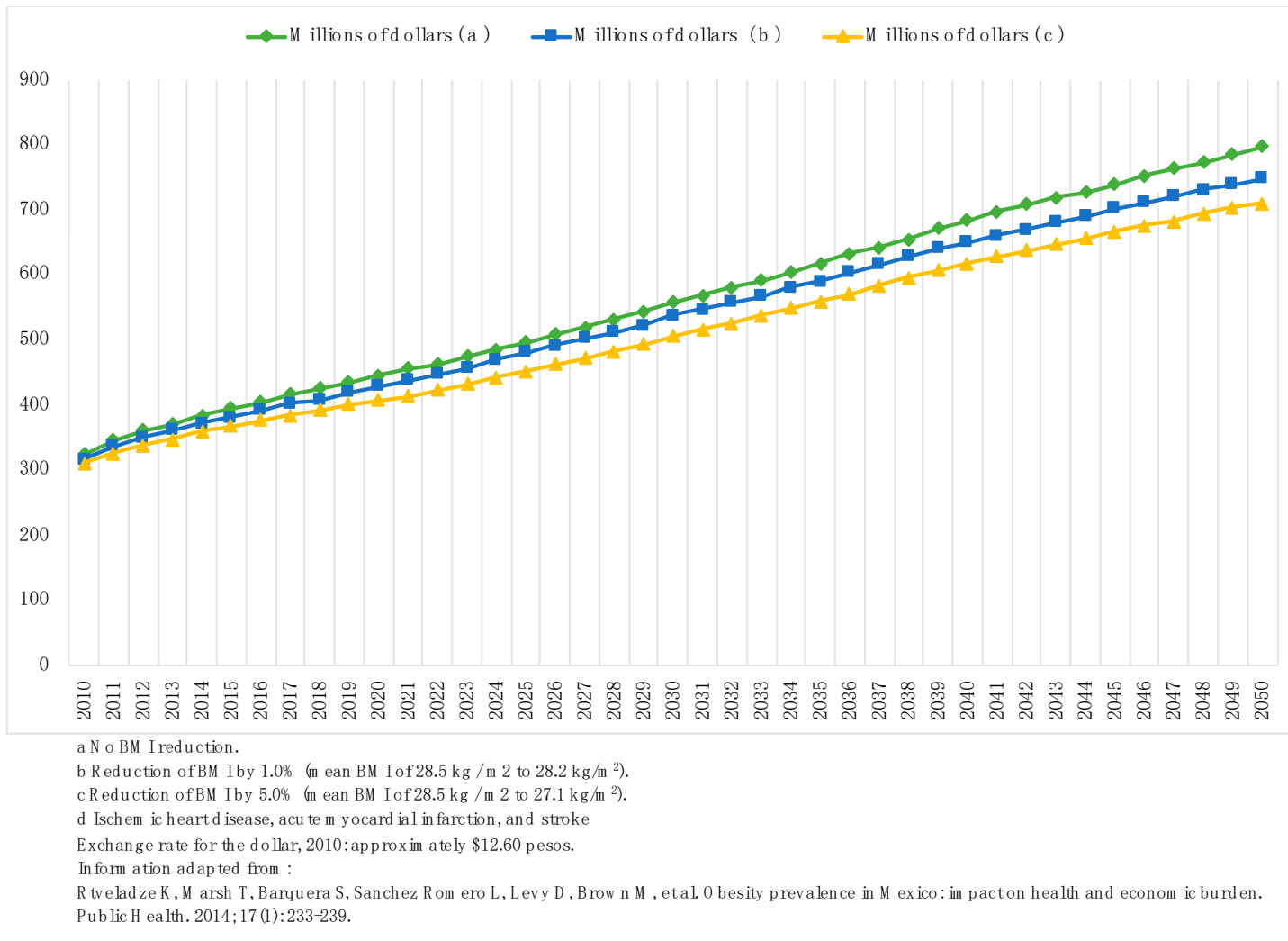

Figure 2. Projected costs of ischemic heart disease, acute myocardial infarction, and stroke. Mexico 2010-2050.

\subsection{Strategies to Decrease the Burden of CVD}

According to the $\mathrm{WHO}$, strategies such as reducing high sodium intake, decreasing tobacco use, and optimization of pharmacological treatment, are the Best Buy Interventions against CVD [3]. An additional investment of USD \$1.5 per capita per year to scale up these strategies between 2015 and 2030, would mean avoiding the incidence of eight million IHD cases and 13 million strokes in countries where the highest chronic disease burden is concentrated [65].

Studies in some mega-countries have shown how beneficial the implementation of actions similar to the Best Buy Interventions could be to the primary prevention of CVD. For instance, in the U.S.A., adherence to a healthy diet and other lifestyle recommendations are associated with a reduction of up to $\approx 92 \%$ of cardiovascular risk in the population [76-78]. In India, a reduction of $3 \mathrm{~g} / \mathrm{d}$ in average population salt consumption through regulations would prevent $\approx 400,000$ and $\approx 81,000$ CVD events and deaths over 30 years, respectively [63]. Unhealthy food taxation is also a promising strategy against CVD. The projections in Mexico indicate that a reduction of $10 \%$ in the consumption of sugar-sweetened beverages through taxes could prevent $\approx 46,300 \mathrm{CHD}$ cases and 14,200 myocardial infarctions over 10 years [73]. In India, a $10 \%$ palm oil tax could reduce $\approx 363,000$ deaths by myocardial infarction in 10 years [64]. Another kind of policy to reduce the CVD burden are the subsidies for healthy food. For example, a $10 \%$ reduction in the prices of fruits, vegetables, and nuts/seeds through this strategy, could prevent $0.6 \%(\approx 2213), 0.8 \%(\approx 2873)$, and $0.9 \%(\approx 3148)$ of the total annual CHD deaths in the U.S.A., respectively [79] (Table 2).

The control of hypercholesterolemia [80], hypertension [81], and diabetes [80], as well as smoking cessation [82] in the secondary prevention level, are essential to promptly reduce the CVD burden [83]. Studies aimed to evaluate the potential of controlling these risk factors have shown substantial expected benefits. For instance, simulation models in the U.S.A. indicate that optimal achievement of management goals of hypertension could reduce $\approx 56,000$ events and $\approx 13,000$ deaths from CVD annually $[75,81]$. In China, projections show that optimizing and broadening lipid-lowering therapy 
coverage could avoid $\approx 850,000$ cases of myocardial infarction and $\approx 300,000$ deaths from CVD each year [54]. In Japan, results derived from a prospective cohort study showed a reduction of $\approx 66 \%$ in the risk of cardiovascular events in healthy adult men associated with smoking cessation for a period equal to or greater than four years [74] (Table 2). However, optimal treatment goals are hard to achieve. In order to effectively treat these conditions, various elements and actions have been recommended: (1) Having a well-trained multidisciplinary health team (e.g., through skills certification); (2) making adjustments to schools' health curriculums (involving the newest treatment guidelines); (3) incorporating family in therapies; (4) creating mutual-help groups with motivation strategies; (5) improving accessibility and availability of medications; and (6) implementing well-structured interventions (e.g., by using electronic health records) [84].

\section{Discussion}

Compared to developed mega-countries, Mexico and the nations with a similar o lesser HDI experience greater challenges related to CVD. In these countries, dietary risk factors contribute more to cardiovascular mortality. Furthermore, there is a greater probability of premature death from CVD and in the last decades, there has been a lower decrease in mortality rates. The economic burden from CVD was significant in both the developing and developed countries analyzed, representing the main health expenditure. Making this comparison was not possible for Nigeria and Brazil since the information was not identified. Additionally, projections indicate that this economic threat will remain significant for these countries by 2030-especially for China, India, and the U.S.A.

The inverse association between countries' development and the burden of chronic diseases has also been described at the sub-national level in various countries [85]. In Southern Mexico, there was a greater increase in mortality from diabetes and CVD at the end of the 20th century in comparison to the northern region, which is more developed $[6,85]$. Because of this, authorities should not only implement a comprehensive strategy, but they should also design targeted strategies that consider the needs of every region. In the southern region, for example, coverage of antihypertensive drugs should be expanded to reduce the high prevalence of adults with hypertension who are not undergoing this treatment [72]. On the other hand, the high prevalence of hypercholesterolemia observed in this study is coupled with low-quality diets that have greater energy density in the northern region [86,87]. This could be mitigated by improving the food environment, dietary recommendations, and increasing availability of and adherence to lipid-lowering treatment in this country area.

This paper gives an overview of the CVD burden in seven mega-countries. Cardiovascular indicators from the GBD study, the WHO Global Health Observatory, national surveys, and from published literature allowed us to compare epidemiological scenarios among nations. Presenting this information, along with CVD economic impact data, provides stakeholders with a comprehensive source of evidence regarding gaps in research, critical epidemiological actions, and financial measures that need to be addressed to counteract CVD. One of the identified barriers in some developing countries is the limited CVD risk monitoring in epidemiological surveillance systems. In Nigeria, for example, there is no representative national data regarding the population's lipid profile [88]. A similar situation occurs in three of the most important national surveys in India [89]. In Brazil, the population's lipid concentration has been only evaluated in a few cities [90] and the assessment of hypercholesterolemia in the National Health Survey is limited to self-reporting [43]. Therefore, governments and academic groups from these countries should secure resources to carry out population level studies that describe the prevalence of dyslipidemias. Prioritizing this would allow for a better design, evaluation, and reformulation of strategies to combat CVD.

The development of hypercholesterolemia, hypertriglyceridemia, and hypoalphalipoproteinemia is associated with the presence of obesity and its lifestyle-related determinants [91-94]. These four CVD risk factors are the most prevalent in Mexico and have been reported to be even greater than in some of the analyzed countries. In Mexico and India, adults with overweight are 5.25 and 4.15 times more likely, respectively, to have mixed dyslipidemias and hypertriglyceridemia $[38,95]$, while up to 
95.2\% of American individuals with obesity have lipid abnormalities [96]. Considering that reductions starting at $1 \%$ of the Mexican population's average BMI would save up to $\$ 986$ million USD in the next years [51], intensive strategies to improve dietary patterns, foster active lifestyles, and increase access to pharmacological treatments that normalize bodyweight and the lipid profile could be vital to moderate costs generated by CVD in Mexico and the other nations.

Statins are the most utilized medications in Mexico to treat CVD due to their cost-effectiveness [97]. Their average unit cost for Mexican health public institutions is $\approx$ USD-PPP \$1 [29]. However, the potential benefit of this treatment for Mexicans' cardiovascular health is mainly hampered by low adherence to medication, as in other countries [98-100]. In addition, low nutritional quality diets $[16,18,101]$ and the high prevalence of physical inactivity and sedentarism $[102,103]$ in the country are major constraints for the statins' positive effects. Inaccurate classification of cardiovascular risk profiles by medical professionals and the lack of diagnosis, are other significant challenges to treatment. This leads to poor management of high-risk patients or extraneous therapies in lower-risk subjects that generate unnecessary costs [104]. This practice, in addition to high prescribed doses of statins and having a high cardiovascular risk profile, have been associated with the non-compliance of the LDL-c goals in Mexico, Brazil, and India [104].

These challenges highlight the need to explore new care and treatment options, especially for population groups with the greatest cardiovascular risk. One alternative includes pharmaceuticals that enhance the receptor activity of the LDL-c molecules and its uptake from plasma to the cells. Until now, these drugs have been indicated in a small proportion of cases and have begun to be studied in clinical randomized control trials $[105,106]$. Nonetheless, due to their approximate cost/person/year of $\$ 14,000$ USD, these pharmaceuticals are not cost-effective in clinical practice [107,108]. Given the high number of individuals in the population who are struggling with the affordability of CVD medicines, including aspirin, $\beta$ blockers, angiotensin-converting enzyme inhibitors, and statins in mega-countries, such as Brazil, China, and India [109], cheaper drugs are still a priority. A lower-cost option is a polypill made from the combination of active substances that produce drug synergism. Its use and formulation with patent-free substances have been proposed as strategies to improve adherence and cost-effectiveness of treatment for CVD [110-118]. However, consistent evidence is needed to support generalized use.

Due to the lack of comparable studies on CVD-related expenditures, the economic burden produced by these diseases cannot be standardized or easily characterized in the analyzed countries. Data from developed countries are obtained through comprehensive economic analyses, but with distinct methodologies and indicators. In estimates from the U.S.A., for example, costs were considered from hospital care, ambulance and emergency room, nursing services, and medications. In contrast, estimates from Japan were obtained from a study which adapted the Cost of Illness method, taking into account direct costs of medical attention, morbidity, mortality, and the long-term costs and economic burden for families [53,59]. In Mexico, four recent studies developed with different comprehensive methods, national registries, and population-based datasets cover economic estimates for hypertension, IHD, heart failure, myocardial infarction, and atrial fibrillation from 2012 to 2017 [44-47]. On the other hand, although a considerable number of studies from India and Nigeria at the regional level focused on catastrophic health expenditures or micro-costing approaches was identified, the nationwide current information on total CVD-related costs was limited. Methodological frameworks of some of these studies were not clearly reported (see Supplementary file 2).

Despite this heterogeneity, it is possible to suggest that the financial burden of CVD in developing countries may be higher than reported if all types of costs were considered. For example, estimates in Mexico indicate that four kinds of these diseases consumed approximately USD-PPP $\$ 11.2$ billion in 2015 or the equivalent to $\approx 4 \%$ of the total health expenses in the country [45]. Although this study, considerably similar to another in Brazil [68], uses direct costs such as patients' hospital care and the loss of economic productivity, these statistics and those from previous years would increase if out-of-pocket expenditures were included, which represent $44 \%$ of the total health expenditure and more than $50 \%$ of total expenses associated with hypertension in Mexican adults [46,47]. Out-of-pocket 
CVD expenses for 15 months in countries such as China and India have been reported to be $15.0 \%-40.1 \%$ and $39.3 \%-54.9 \%$ of annual household expenses, respectively [119]. In addition, catastrophic health expenditure in some of the analyzed mega-countries is considerably higher in households where family members with hypertension live [120]; further, it is significantly high, even for insured individuals [121]. In any case, the financial stress due to CVD experienced by families should be considered in future comprehensive studies.

Management of CVD is a large-scale challenge. In 2006, $\approx 60 \%$ of Mexican adults required interventions to reduce their cardiovascular risk [31]. Indeed, this prevalence could be greater considering current population ageing trends and CVD treatment updated guidelines. Optimal treatment for current CVD cases is key for diminishing the cardiovascular burden in the short term [84]. However, deficiencies in health systems, the complexity of illness, and the high cost of secondary care $[84,122]$ make it difficult to effectively treat CVD in countries such as Mexico [84]. Because of this, authorities from each country should opt to allocate resources to a systematic strategy focused on improving capacity of health care personnel through modifications to university study plans that encourage of lifelong learning, strengthening family inclusion and motivation techniques in medical care, and on guaranteeing technological infrastructure and drugs for adequate management and treatment of patients.

The CVD burden should also be addressed through effective interventions based on the life-course approach and environmental strategies. The modification of the food system through taxes to sugar sweetened beverages (SSBs), an understandable front-of-pack nutrition label, and subsidies for healthy foods are complementary components to prevent cardiovascular risk factors, CVD, and its comorbidities in the next generations. They favor healthy diets and facilitate informed consumption decisions in general and at-risk population $[3,123,124]$. Furthermore, the SSBs tax has been shown to reduce sales of these products in Mexico and other countries [125-130], which in the long-term could decrease cardiovascular risk factors such as high BMI and hyperglycemia, bringing considerable economic benefits. Accordingly, this fiscal measure should be considered a policy with great potential if it is adopted as a CVD prevention strategy.

\section{Conclusions}

The findings of this study have implications for future research and public health actions related to CVD in the analyzed countries. In general, this study highlights for international congruency in methods and implementation practices in order to more effectively compare across countries, specifically to identify the attributable economic impact of CVD in these nations, considering expenditures absorbed by institutions and families. Public health implications are evident considering that CVD currently generate a significant health and economic burden around the world, particularly in Mexico and six other mega-countries that hold approximately $50 \%$ of the global population. Although the increase of morbidity and cardiovascular mortality in the developed countries from this group of nations has slowed in the last years, projections show that costs related to CVD will remain significant in the next decades. Consequently, investment in strategies and prevention policies must continue and be raised in order to reduce the incidence of these diseases and their risk factors in developed mega-countries.

Developing mega-countries face greater obstacles due to CVD than developed countries. They also experience shortcomings in clinical practice associated with failure to reach lipid profile targets and deficiencies in epidemiological surveillance instruments that limit cardiovascular monitoring in the population. This suggests an urgent need to implement well-structured interventions at the primary and secondary care level in order to optimize diagnosis, the prescription of pharmacological treatment and lifestyles, and the adherence to these interventions, especially in countries such as Mexico and India, where higher prevalence of some dyslipidemias are observed. The evaluation of dyslipidemias in the Nigerian and Brazilian population through national representative studies must be strengthened. Governments from developing mega-countries must centralize their efforts so that the food system does not favor the obesogenic environment and exacerbate the high contribution that dietary risk 
factors already have on CVD mortality rates, as this would translate into a substantial reduction in the economic impact and health burden caused by these diseases in the next generations. The loss of sustainable development that CVD would eventually cause to the most populated countries in the world will only be mitigated through the implementation of cost-effective strategies at all the medical care levels and by adopting a preventive health approach.

Supplementary Materials: The following are available online at http://www.mdpi.com/1660-4601/16/20/ 4041/s1, PRISMA checklist (see Supplementary file 1), Supplementary Table S1 (see Supplementary file 2), and Supplementary Tables S2-S5 (see Supplementary file 2).

Author Contributions: Conceptualization: K.M.-H., A.P.-T., C.H.-A., S.B. Formal Analysis: K.M.-H., A.P.-T., L. Á.-B., C.A.A.-S., S.B. Funding Acquisition: S.B., C.A.A.-S. Project Administration: K.M.-H., C.H.-A. Writing—original draft: K.M.-H., A.P.-T., S.B. Writing—review and editing: K.M.-H., L.Á.-B., C.A.A.-S., S.B. All authors read and approved the final manuscript.

Funding: Development of research in this article was supported by the financing provided to the Mexican National Institute of Public Health (Spanish acronym: INSP) by Sanofi under registration number DIREGL07857 (S.B.); the Fogarty International Center of the National Institutes of Health (http://www.fic.nih.gov/Pages/Default.aspx) under Award Number R03TW009061 (S.B., A.P.-T.). Also is supported in part by the Robert Wood Johnson Foundation grant under Award Number 74155 (C.H.-A., K.M.-H.). The funders had no role in study design, data collection and analysis, decision to publish, or preparation of the manuscript.

Acknowledgments: The authors thank Mariel White for her technical contribution in this work.

Conflicts of Interest: The authors have declared that they have no competing interests.

\section{References}

1. Global, regional, and national age-sex specific all-cause and cause-specific mortality for 240 causes of death, 1990-2013: A systematic analysis for the Global Burden of Disease Study 2013. Lancet 2015, 385, 117-171. [CrossRef]

2. Ávila-Burgos, L.; Cahuana-Hurtado, L.; González-Domínguez, D.; Aracena-Genao, B.; MontañezHernández, J.C.; Serván-Mori, E.E.; RiveraPeña, G. Cuentas en Diabetes Mellitus, Enfermedades Cardiovasculares y Obesidad, México 2006; Instit: Ciudad de México Cuernavaca, Mexico, 2006.

3. WHO. Global Action Plan for the Prevention and Control of Noncommunicable Diseases 2013-2020; World Health Organization: Geneva, Switzerland, 2013; Available online: http://apps.who.int/iris/bitstream/10665/94384/1/ 9789241506236_eng.pdf (accessed on 19 October 2019).

4. Bloom, D.E.; Cafiero, E.; Jané-Llopis, E.; Abrahams-Gessel, S.; Bloom, L.R.; Fathima, S.; Feigl, A.B.; Gaziano, T.; Mowafi, M.; Pandya, A.; et al. The Global Economic Burden of Noncommunicable Diseases; Program on the Global Demography of Aging: Cambridge, MA, USA, 2012.

5. Laslett, L.J.; Alagona, P.; Clark, B.A.; Drozda, J.P.; Saldivar, F.; Wilson, S.R.; Poe, C.; Hart, M. The worldwide environment of cardiovascular disease: Prevalence, diagnosis, therapy, and policy issues: A report from the american college of cardiology. J. Am. Coll. Cardiol. 2012, 60, S1-S49. [CrossRef] [PubMed]

6. Barquera, S.; Tovar-Guzmán, V.; Campos-Nonato, I.; González-Villalpando, C.; Rivera-Dommarco, J. Geography of diabetes mellitus mortality in Mexico: An epidemiologic transition analysis. Arch. Med. Res. 2003, 34, 407-414. [CrossRef]

7. Villalpando, S.; Shamah-Levy, T.; Rojas, R.; Aguilar-Salinas, C.A. Trends for type 2 diabetes and other cardiovascular risk factors in Mexico from 1993-2006. Salud Publ. Mex. 2010, 52, S72-S79. [CrossRef]

8. Barquera, S.; Campos-Nonato, I.; Hernández-Barrera, L.; Pedroza, A.; Rivera-Dommarco, J.a. Prevalencia de obesidad en adultos mexicanos 2000-2012. Salud Publ. Mex. 2013, 55, 151-160. [CrossRef]

9. Aguilar-Salinas, C.A.; Gomez-Perez, F.J.; Rull, J.; Villalpando, S.; Barquera, S.; Rojas, R. Prevalence of dyslipidemias in the Mexican National Health and Nutrition Survey 2006. Salud Publ. Mex. 2010, 52, S44-S53. [CrossRef]

10. Institute for Health Metrics and Evaluation (IHME). GBD Compare Data Visualization; IHME: Seattle, WA, USA; University of Washington: Washington, DC, USA, 2016; Available online: http://vizhub.healthdata.org/ gbd-compare (accessed on 20 January 2018).

11. Aikawa, M.; Libby, P. The vulnerable atherosclerotic plaque: Pathogenesis and therapeutic approach. Cardiovasc. Pathol. 2004, 13, 125-138. [CrossRef] 
12. Barquera, S.; Pedroza-Tobias, A.; Medina, C. Cardiovascular diseases in mega-countries. Curr. Opin. Lipidol. 2016, 27, 329-344. [CrossRef]

13. Barquera, S.; Pedroza-Tobías, A.; Medina, C.; Hernández-Barrera, L.; Bibbins-Domingo, K.; Lozano, R.; Moran, A.E. Global Overview of the Epidemiology of Atherosclerotic Cardiovascular Disease. Arch. Med. Res. 2015, 46, 328-338. [CrossRef]

14. Rivera, J.A.; Barquera, S.; Campirano, F.; Campos, I.; Safdie, M.; Tovar, V. Epidemiological and nutritional transition in Mexico: Rapid increase of non-communicable chronic diseases and obesity. Public Health Nutr. 2002, 5, 113-122. [CrossRef]

15. Stevens, G.; Dias, R.H.; Thomas, K.J.A.; Rivera, J.A.; Carvalho, N.; Barquera, S.; Hill, K.; Ezzati, M. Characterizing the epidemiological transition in Mexico: National and subnational burden of diseases, injuries, and risk factors. PLoS Med. 2008, 5, 0900-0910.

16. Stern, D.; Piernas, C.; Barquera, S.; Rivera, J.A.; Popkin, B.M. Caloric beverages were major sources of energy among children and adults in Mexico, 1999-2012. J. Nutr. 2014, 144, 949-956. [CrossRef] [PubMed]

17. Barquera, S.; Campirano, F.; Bonvecchio, A.; Hernandez-Barrera, L.; Rivera, J.A.; Popkin, B.M. Caloric beverage consumption patterns in Mexican children. Nutr. J. 2010, 9, 47. [CrossRef] [PubMed]

18. Barquera, S.; Hernandez-Barrera, L.; Tolentino, M.L.; Espinosa, J.; Ng, S.W.; Rivera, J.A.; Popkin, B.M. Energy intake from beverages is increasing among Mexican adolescents and adults. J. Nutr. 2008, 138, 2454-2461. [CrossRef] [PubMed]

19. Flores, M.; Macias, N.; Rivera, M.; Lozada, A.; Barquera, S.; Rivera-Dommarco, J.; Tucker, K.L. Dietary patterns in Mexican adults are associated with risk of being overweight or obese. J. Nutr. 2010, 140, 1869-1873. [CrossRef]

20. Barquera, S.; Campos-Nonato, I.; Hernandez-Barrera, L.; Villalpando, S.; Rodriguez-Gilabert, C.; Durazo-Arvizu, R.; Aguilar-Salinas, C.A. Hypertension in Mexican adults: Results from the National Health and Nutrition Survey 2006. Salud Publ. Mex. 2010, 52, S63-S71. [CrossRef]

21. Popkin, B.M. Nutrition Transition and the Global Diabetes Epidemic. Curr. Diab. Rep. 2015, 15, 64. [CrossRef]

22. Douglas, K.A.; Yang, G.; McQueen, D.V.; Puska, P. Mega Country Health Promotion Network Surveillance Initiative. In Global Behavioral Risk Factor Surveillance; Douglas, K.A., Yang, G., McQueen, D.V., Puska, P., Eds.; Springer: Boston, MA, USA, 2003; pp. 179-196.

23. Kennedy, E. Healthy lifestyles. healthy people-The Mega Country Health Promotion Network. Asia Pac. J. Clin. Nutr. 2002, 11, S738-S739. [CrossRef]

24. Stewart, F. Capabilities and Human Development: Beyond the individual-the critical role of social institutions and social competencies. Hum. Dev. Rep.-UNDP 2013, 3, 1-20.

25. Moher, D.; Liberati, A.; Tetzlaff, J.; Altman, D.G.; Altman, D.; Antes, G. Preferred reporting items for systematic reviews and meta-analyses: The PRISMA statement (Chinese edition). J. Chin. Integr. Med. 2009, 7, 889-896. [CrossRef]

26. Liberati, A.; Altman, D.G.; Tetzlaff, J.; Mulrow, C.; Gøtzsche, P.C.; Ioannidis, J.P.A.; Clarke, M.; Devereaux, P.J.; Kleijnen, J.; Moher, D. The PRISMA statement for reporting systematic reviews and meta-analyses of studies that evaluate health care interventions: Explanation and elaboration. J. Clin. Epidemiol. 2009, 62, e1-e34. [CrossRef] [PubMed]

27. Olaiz-Fernández, G.; Rivera-Dommarco, J.; Shamah-Levy, T.; Rojas, R.; Villalpando Hernández, S.; Hernández-Avila, M.; Sepúlveda-Amor, J. Encuesta Nacional de Salud y Nutrición 2006; Instituto Nacional de Salud Pública: Cuernavaca, Mexico, 2006.

28. Gutiérrez, J.P.; Rivera-Dommarco, J.; Shamah-Levy, T.; Villalpando-Hernández, S.; Franco, A.; Cuevas-Nasu, L.; Romero-Martínez, M.; Hernández-Ávila, M. Encuesta Nacional de Salud y Nutrición 2012; Resultados Nacionales; Instituto Nacional de Salud Pública: Cuernavaca, Mexico, 2012.

29. Instituo Mexicano del Seguro Social (IMSS). Portal de compras del IMSS. Available online: http://compras. imss.gob.mx/ (accessed on 9 July 2018).

30. Organization, W.H. Global Health Observatory Data Repository; World Health Organization: Geneva, Switzerland, 2013.

31. Gomez-Perez, F.J.; Rojas, R.; Villalpando, S.; Barquera, S.; Rull, J.; Aguilar-Salinas, C.A. Prevention of cardiovascular disease based on lipid lowering treatment: A challenge for the Mexican health system. Salud Publ. Mex. 2010, 52, S54-S62. [CrossRef] 
32. Executive Summary of The Third Report of The National Cholesterol Education Program (NCEP) Expert Panel on Detection, Evaluation, and Treatment of High Blood Cholesterol In Adults (Adult Treatment Panel III). JAMA 2001, 285, 2486-2497. [CrossRef] [PubMed]

33. Carroll, M.D.; Fryar, C.D.; Nguyen, D.T. Key findings Data from the National Health and Nutrition Examination Survey. In Total and High-density Lipoprotein Cholesterol in Adults: United States, 2015-2016; Centers for Disease Control and Prevention: Washington, DC, USA, 2015. Available online: https: //www.cdc.gov/nchs/data/databriefs/db290_table.pdf\#2 (accessed on 19 October 2019).

34. Toth, P.P.; Potter, D.; Ming, E.E. Prevalence of lipid abnormalities in the United States: The National Health and Nutrition Examination Survey 2003-2006. J. Clin. Lipidol. 2012, 6, 325-330. [CrossRef] [PubMed]

35. Kuklina, E.V.; Carroll, M.D.; Shaw, K.M.; Hirsch, R. Trends in high LDL cholesterol, cholesterol-lowering medication use, and dietary saturated-fat intake: United States, 1976-2010. NCHS Data Brief. 2013, 117, 1-8.

36. National Health and Nutrition Survey in Japan. Ministry of Health, Labour, and Welfare. 2015. Available online: http://www.mhlw.go.jp/toukeiitiran/gaiyo/k-eisei.html (accessed on 19 October 2019).

37. Lin, C.F.; Chang, Y.H.; Chien, S.C.; Lin, Y.H.; Yeh, H.Y. Epidemiology of Dyslipidemia in the Asia Pacific Region. Int. J. Gerontol. 2018, 12, 2-6. [CrossRef]

38. Joshi, S.R.; Anjana, R.M.; Deepa, M.; Pradeepa, R.; Bhansali, A.; Dhandania, V.K.; Joshi, P.P.; Unnikrishnan, R.; Nirmal, E.; Subashini, R.; et al. Prevalence of dyslipidemia in urban and rural India: The ICMR-INDIAB study. PLoS ONE 2014, 9, e96808. [CrossRef]

39. Oguoma, V.M.; Nwose, E.U.; Ulasi, I.I.; Akintunde, A.A.; Chukwukelu, E.E.; Bwititi, P.T.; Richards, R.S.; Skinner, T.C. Cardiovascular disease risk factors in a Nigerian population with impaired fasting blood glucose level and diabetes mellitus. BMC Public Health 2017, 17, 36. [CrossRef]

40. Huang, Y.; Gao, L.; Xie, X.; Tan, S.C. Epidemiology of dyslipidemia in Chinese adults: Meta-analysis of prevalence, awareness, treatment, and control. Popul. Health Metr. 2014, 12, 28. [CrossRef]

41. Zhang, M.; Deng, Q.; Wang, L.; Huang, Z.; Zhou, M.; Li, Y.; Zhao, Z.; Zhang, Y.; Wang, L. Prevalence of dyslipidemia and achievement of low-density lipoprotein cholesterol targets in Chinese adults: A nationally representative survey of 163,641 adults. Int. J. Cardiol. 2018, 260, 196-203. [CrossRef]

42. Santos, R.D.; Bensenor, I.M.; Pereira, A.C.; Lotufo, P.A. Dyslipidemia according to gender and race: The Brazilian Longitudinal Study of Adult Health (ELSA-Brasil). J. Clin. Lipidol. 2016, 10, 1362-1368. [CrossRef] [PubMed]

43. Lotufo, P.A.; Santos, R.D.; Sposito, A.C.; Bertolami, M.; Rocha-Faria Neto, J.; Izar, M.C.; Szwarcwald, C.; Prado, R.R.; Stoppa, S.R.; Malta, D.C.; et al. Self-Reported High-Cholesterol Prevalence in the Brazilian Population: Analysis of the 2013 National Health Survey. Arq. Bras. Cardiol. 2017, 108, 411-416. [CrossRef] [PubMed]

44. Figueroa-Lara, A.; Gonzalez-Block, M.A.; Alarcon-Irigoyen, J. Medical Expenditure for Chronic Diseases in Mexico: The Case of Selected Diagnoses Treated by the Largest Care Providers. PLoS ONE 2016, 11, e0145177. [CrossRef] [PubMed]

45. Stevens, B.; Pezzullo, L.; Verdian, L.; Tomlinson, J.; Estrada-Aguilar, C.; George, A.; Verdejo-París, J. The economic burden of hypertension, heart failure, myocardial infarction, and atrial fibrillation in Mexico. Arch. Cardiol. Mex. 2018, 88, 241. [CrossRef]

46. Arredondo, A.; Cuadra, S.M.; Duarte, M.B. Challenges of the epidemiological and economic burdens associated with hypertension in middle income countries: Evidence from Mexico Chronic Disease epidemiology. BMC Public Health 2015, 15, 1106. [CrossRef]

47. Arredondo, A.; Duarte, M.B.; Cuadra, S.M. Epidemiological and financial indicators of hypertension in older adults in Mexico: Challenges for health planning and management in Latin America. Int. J. Health Plan. Manag. 2017, 32, e121-e136. [CrossRef]

48. Butland, B.; Jebb, S.; Kopelman, P.; McPherson, K.; Thomas, S.; Mardell, J.; Parry, V. Foresight Tackling Obesities: Future Choices-Project Report. 2007; Government Office for Science: London, UK, 2007.

49. Kopelman, P.; Jebb, S.A.; Butland, B. Executive summary: Foresight: “Tackling Obesities: Future Choices" project. Obes Rev. 2007, 8, vi-ix.

50. McPherson, K.; Marsh, T.; Foresight, B.M. Tackling Obesities: Future Choices-Modelling Future Trends in Obesity $\mathcal{E}$ Their Impact on Health; Government Office for Science: London, UK, 2007. 
51. Rtveladze, K.; Marsh, T.; Barquera, S.; Sanchez Romero, L.M.; Levy, D.; Melendez, G.; Webber, L.; Kilpi, F.; McPherson, K.; Brown, M. Obesity prevalence in Mexico: Impact on health and economic burden. Public Health Nutr. 2014, 17, 233-239. [CrossRef]

52. Stam-Slob, M.C.; van der Graaf, Y.; Greving, J.P.; Dorresteijn, J.A.N.; Visseren, F.L.J. Cost-Effectiveness of Intensifying Lipid-Lowering Therapy With Statins Based on Individual Absolute Benefit in Coronary Artery Disease Patients. J. Am. Heart Assoc. 2017, 6, e004648. [CrossRef]

53. Dieleman, J.L.; Baral, R.; Birger, M.; Bui, A.L.; Bulchis, A.; Chapin, A.; Hamavid, H.; Horst, C.; Johnson, E.K.; Joseph, J.; et al. US Spending on Personal Health Care and Public Health, 1996-2013. JAMA 2016, 316, 2627-2646. [CrossRef]

54. Stevens, W.; Peneva, D.; Li, J.Z.; Liu, L.Z.; Liu, G.; Gao, R.; Lakdawalla, D.N. Estimating the future burden of cardiovascular disease and the value of lipid and blood pressure control therapies in China. BMC Health Serv. Res. 2016, 16, 175. [CrossRef] [PubMed]

55. Benjamin, E.J.; Blaha, M.J.; Chiuve, S.E.; Cushman, M. Heart Disease and Stroke Statistics-2017 Update. Circulation 2017, 135, e146-e603. [CrossRef] [PubMed]

56. Levi, J.; Segal, L.M.; Juliano, C. Prevention for a Healthier America: Investments in Disease Prevention Yield Significant Savings, Stronger Communities; Trust for America's Health: Washington, DC, USA, 2008.

57. Chen, W.-W.; Gao, R.-L.; Liu, L.-S.; Zhu, M.-L.; Wang, W.; Wang, Y.-J.; WU, Z.-S.; LI, H.-J.; GU, D.-F.; YANG, Y.-J.; et al. China cardiovascular diseases report 2015: A summary. J. Geriatr. Cardiol. 2017, 14, 1-10. [PubMed]

58. Wang, S.; Marquez, P.; Langenbrunner, J.; Niessen, L.; Suhrcke, M.; Song, F. Toward a Healthy and Harmonious Life in China: Stemming the Rising Tide of Non-Communicable Diseases; Washingt World Bank: Washington, DC, USA, 2011.

59. Matsumoto, K.; Hanaoka, S.; Wu, Y.; Hasegawa, T. Comprehensive Cost of Illness of Three Major Diseases in Japan. J. Stroke Cerebrovasc. Dis. 2017, 26, 1934-1940. [CrossRef] [PubMed]

60. Siqueira, A.S.E.; Siqueira-Filho, A.G.; Land, M.G.P. Analysis of the Economic Impact of Cardiovascular Diseases in the Last Five Years in Brazil. Arq. Bras. Cardiol. 2017, 109, 39-46. [CrossRef] [PubMed]

61. Abegunde, D.O.; Mathers, C.D.; Adam, T.; Ortegon, M.; Strong, K. The burden and costs of chronic diseases in low-income and middle-income countries. Lancet 2007, 370, 1929-1938. [CrossRef]

62. Birabi, B.N.; Oke, K.I.; Dienye, P.O.; Okafor, U.C. Cost burden of post stroke condition in Nigeria: A pilot study. Glob. J. Health Sci. 2012, 4, 17. [CrossRef]

63. Basu, S.; Stuckler, D.; Vellakkal, S.; Ebrahim, S. Dietary salt reduction and cardiovascular disease rates in India: A mathematical model. PLoS ONE 2012, 7, e44037. [CrossRef]

64. Basu, S.; Babiarz, K.S.; Ebrahim, S.; Vellakkal, S.; Stuckler, D.; Goldhaber-Fiebert, J.D. Palm oil taxes and cardiovascular disease mortality in India: Economic-epidemiologic model. BMJ 2013, 347, f6048. [CrossRef]

65. Bertram, M.Y.; Sweeny, K.; Lauer, J.A.; Chisholm, D.; Sheehan, P.; Rasmussen, B.; Raj-Upreti, S.; PrasaiDixit, L.; George, K.; Deane, S. Investing in non-communicable diseases: An estimation of the return on investment for prevention and treatment services. Lancet 2018, 391, 2071-2078. [CrossRef]

66. Bloom, D.E.; Cafiero-Fonseca, E.T.; McGovern, M.E.; Prettner, K.; Stanciole, A.; Weiss, J.; Bakkila, S.; Rosenberg, L. The macroeconomic impact of non-communicable diseases in China and India: Estimates, projections, and comparisons. J. Econ. Ageing 2014, 4, 100-111. [CrossRef]

67. Bloom, D.E.; Chen, S.; Kuhn, M.; McGovern, M.E.; Oxley, L.; Prettner, K. The economic burden of chronic diseases: Estimates and projections for China, Japan, and South Korea. J. Econ. Ageing 2018, 26, 100163. [CrossRef]

68. Stevens, B.; Pezzullo, L.; Verdian, L.; Tomlinson, J.; George, A.; Bacal, F. The Economic Burden of Heart Conditions in Brazil. Arq. Bras. Cardiol. 2018, 111, 29-36. [CrossRef] [PubMed]

69. Association, A.H. Cardiovascular Disease: A Costly Burden for America Projections through 2035; American Heart Association: Dallas, TX, USA, 2017.

70. Dunbar, S.B.; Khavjou, O.A.; Bakas, T.; Hunt, G.; Kirch, R.A.; Leib, A.R.; Morrison, R.S.; Poehler, D.C.; Roger, V.L.; Whitsel, L.P.; et al. Projected Costs of Informal Caregiving for Cardiovascular Disease: 2015 to 2035: A Policy Statement From the American Heart Association. Circulation 2018, 137, e558-e577. [CrossRef]

71. Gochi, T.; Matsumoto, K.; Amin, R.; Kitazawa, T.; Seto, K.; Hasegawa, T. Cost of illness of ischemic heart disease in Japan: A time trend and future projections. Environ. Health Prev. Med. 2018, 23, 1-7. [CrossRef] 
72. Campos-Nonato, I.; Hernández-Barrera, L.; Pedroza-Tobías, A.; Medina, C.; Barquera, S. Hipertensión arterial en adultos mexicanos: Prevalencia, diagnóstico y tipo de tratamiento. Ensanut MC 2016. Salud Publ. Mex. 2018, 60, 233. [CrossRef]

73. Sánchez-Romero, L.M.; Penko, J.; Coxson, P.G.; Fernández, A.; Mason, A.; Moran, A.E.; Ávila-Burgos, L.; Odden, M.; Barquera, S.; Bibbins-Domingo, K. Projected Impact of Mexico's Sugar-Sweetened Beverage Tax Policy on Diabetes and Cardiovascular Disease: A Modeling Study. PLoS Med. 2016, 13, 1-17. [CrossRef]

74. Kondo, T.; Osugi, S.; Shimokata, K.; Honjo, H.; Morita, Y.; Maeda, K.; Yamashita, K.; Muramatsu, T.; Shintani, S.; Matsushita, K.; et al. Smoking and Smoking Cessation in Relation to All-Cause Mortality and Cardiovascular Events in 25,464 Healthy Male Japanese Workers. Circ. J. 2011, 75, 2885-2892. [CrossRef]

75. Moran, A.E.; Odden, M.C.; Thanataveerat, A.; Tzong, K.Y.; Rasmussen, P.W.; Guzman, D.; Williams, L.; Bibbins-Domingo, K.; Coxson, P.G.; Goldman, L. Cost-Effectiveness of Hypertension Therapy According to 2014 Guidelines. N. Engl. J. Med. 2015, 372, 447-455. [CrossRef]

76. Chomistek, A.K.; Chiuve, S.E.; Eliassen, A.H.; Mukamal, K.J.; Willett, W.C.; Rimm, E.B. Healthy lifestyle in the primordial prevention of cardiovascular disease among young women. J. Am. Coll. Cardiol. 2015, 65, 43-51. [CrossRef]

77. Stampfer, M.J.; Hu, F.B.; Manson, J.E.; Rimm, E.B.; Willett, W.C. Primary prevention of coronary heart disease in women through diet and lifestyle. N. Engl. J. Med. 2000, 343, 16-22. [CrossRef] [PubMed]

78. Chiuve, S.E.; Rexrode, K.M.; Spiegelman, D.; Logroscino, G.; Manson, J.E.; Rimm, E.B. Primary prevention of stroke by healthy lifestyle. Circulation 2008, 118, 947-954. [CrossRef] [PubMed]

79. Peñalvo, J.L.; Cudhea, F.; Micha, R.; Rehm, C.D.; Afshin, A.; Whitsel, L.; Wilde, P.; Gaziano, T.; Pearson-Stuttard, J.; O'Flaherty, M.; et al. The potential impact of food taxes and subsidies on cardiovascular disease and diabetes burden and disparities in the United States. BMC Med. 2017, 15, 1-13. [CrossRef] [PubMed]

80. Stone, N.J.; Robinson, J.G.; Lichtenstein, A.H.; Bairey Merz, C.N.; Blum, C.B.; Eckel, R.H.; Goldberg, A.C.; Gordon, D.; Levy, D.; Lloyd-Jones, D.M.; et al. 2013 ACC/AHA guideline on the treatment of blood cholesterol to reduce atherosclerotic cardiovascular risk in adults: A report of the American College of Cardiology/American Heart Association Task Force on Practice Guidelines. J. Am. Coll. Cardiol. 2014, 63, 2889-2934. [CrossRef] [PubMed]

81. James, P.A.; Oparil, S.; Carter, B.L.; Cushman, W.C.; Dennison-Himmelfarb, C.; Handler, J.; Lackland, D.T.; LeFevre, M.L.; MacKenzie, T.D.; Ogedegbe, O.; et al. 2014 evidence-based guideline for the management of high blood pressure in adults: Report from the panel members appointed to the Eighth Joint National Committee (JNC 8). JAMA 2014, 311, 507-520. [CrossRef] [PubMed]

82. Ockene, I.S.; Miller, N.H. Cigarette smoking, cardiovascular disease, and stroke: A statement for healthcare professionals from the American Heart Association. American Heart Association Task Force on Risk Reduction. Circulation 1997, 96, 3243-3247. [CrossRef]

83. The CDC Diabetes Cost-effectiveness Group. Cost-effectiveness of Intensive Glycemic Control, Intensified Hypertension Control, and Serum Cholesterol Level Reduction for Type 2 Diabetes. JAMA 2002, 287, 2542. [CrossRef]

84. Córdova-Villalobos, J.A.; Barriguete-Meléndez, J.A.; Lara-Esqueda, A.; Barquera, S.; Rosas-Peralta, M.; Hernández-Avila, M.; de León-May, M.E.; Aguilar-Salinas, C.A. Chronic non-communicable diseases in Mexico: Epidemiologic synopsis and integral prevention. Salud Publ. Mex. 2008, 50, 419-427. [CrossRef]

85. Rivera, J.; Barquera, S.; González-Cossío, T.; Olaiz, G.; Sepúlveda, J. Nutrition transition in Mexico and in other Latin American countries. Nutr. Rev. 2004, 62, S149-S157. [CrossRef]

86. Marron-Ponce, J.A.; Sanchez-Pimienta, T.G.; Louzada, M.L.D.C.; Batis, C. Energy contribution of NOVA food groups and sociodemographic determinants of ultra-processed food consumption in the Mexican population. Public Health Nutr. 2018, 21, 87-93. [CrossRef]

87. Ponce, X.; Rodriguez-Ramirez, S.; Mundo-Rosas, V.; Shamah, T.; Barquera, S.; Gonzalez de Cossio, T. Dietary quality indices vary with sociodemographic variables and anthropometric status among Mexican adults: A cross-sectional study. Results from the 2006 National Health and Nutrition Survey. Public Health Nutr. 2014, 17, 1717-1728. [CrossRef] [PubMed]

88. National Population Commission-NPC/Nigeria and ICF International. Demographic and Health Survey 2013; DHS: Abuja, Nigeria, 2013. 
89. Dandona, R.; Pandey, A.; Dandona, L. A review of national health surveys in India. World Heal. Organ. Bull. 2016, 94, 286-296. [CrossRef] [PubMed]

90. Garcez, M.R.; Pereira, J.L.; Fontanelli, M.M.; Marchioni, D.M.L.; Fisberg, R.M. Prevalence of Dyslipidemia According to the Nutritional Status in a Representative Sample of São Paulo. Arq. Bras. Cardiol. 2014, 476-484. [CrossRef] [PubMed]

91. Franssen, R.; Monajemi, H.; Stroes, E.S.G.; Kastelein, J.J.P. Obesity and dyslipidemia. Med. Clin. N. Am. 2011, 95, 893-902. [CrossRef] [PubMed]

92. Klop, B.; Elte, J.W.F.; Cabezas, M.C. Dyslipidemia in obesity: Mechanisms and potential targets. Nutrients 2013, 5, 1218-1240. [CrossRef]

93. Castelli, W. Lipoproteins and cardiovascular disease: Biological basis and epidemiological studies. Value Heal. J. Int. Soc. Pharmacoeconomics Outcomes Res. 1998, 1, 105-109. [CrossRef]

94. Hruby, A.; Hu, F.B. The Epidemiology of Obesity: A Big Picture. Pharmacoeconomics 2015, 33, 673-689. [CrossRef]

95. Barquera, S.; Flores, M.; Olaiz-Fernández, G.; Monterrubio, E.; Villalpando, S.; González, C.; Rivera, J.A.; Sepúlveda, J. Dyslipidemias and obesity in Mexico. Salud Publ. Mex. 2007, 49, s338-s347. [CrossRef]

96. Guo, F.; Garvey, W.T. Trends in Cardiovascular Health Metrics in Obese Adults: National Health and Nutrition Examination Survey (NHANES), 1988-2014. J. Am. Heart Assoc. 2016, 5, e003619. [CrossRef]

97. Mitchell, A.P.; Simpson, R.J. Statin cost effectiveness in primary prevention: A systematic review of the recent cost-effectiveness literature in the United States. BMC Res. Notes 2012, 5, 373. [CrossRef]

98. Berra, K. Lipid-lowering therapy today: Treating the high-risk cardiovascular patient. J. Cardiovasc. Nurs. 2008, 23, 414-421. [CrossRef] [PubMed]

99. Schwiesow, S.J.; Nappi, J.M.; Ragucci, K.R. Assessment of compliance with lipid guidelines in an academic medical center. Ann. Pharmacother. 2006, 40, 27-31. [CrossRef] [PubMed]

100. Zafrir, B.; Cohen, S. Primary prevention in high-risk dyslipidemic patients without an established cardiovascular disease: Undertreatment and rationale for lipid-lowering therapy. Eur. J. Intern. Med. 2006, 17, 495-499. [CrossRef] [PubMed]

101. Rivera, J.A.; Pedraza, L.S.; Aburto, T.C.; Batis, C.; Sanchez-Pimienta, T.G.; Gonzalez de Cosio, T.; López-Olmedo, N.; Pedroza-Tobías, A. Overview of the Dietary Intakes of the Mexican Population: Results from the National Health and Nutrition Survey 2012. J. Nutr. 2016, 146, 1851S-1855S. [CrossRef] [PubMed]

102. Medina, C.; Janssen, I.; Campos, I.; Barquera, S. Physical inactivity prevalence and trends among Mexican adults: Results from the National Health and Nutrition Survey (ENSANUT) 2006 and 2012. BMC Public Health 2013, 13, 1063. [CrossRef] [PubMed]

103. Medina, C.; Tolentino-Mayo, L.; Lopez-Ridaura, R.; Barquera, S. Evidence of increasing sedentarism in Mexico City during the last decade: Sitting time prevalence, trends, and associations with obesity and diabetes. PLoS ONE 2017, 12, e0188518. [CrossRef]

104. Danchin, N.; Almahmeed, W.; Al-Rasadi, K.; Azuri, J.; Berrah, A.; Cuneo, C.A.; Karpov, Y.; Kaul, U.; Kayıkçığlu, M.; Mitchenko, O.; et al. Achievement of low-density lipoprotein cholesterol goals in 18 countries outside Western Europe: The International ChoLesterol management Practice Study (ICLPS). Eur. J. Prev. Cardiol. 2018, 25, 1087-1094. [CrossRef]

105. Sabatine, M.S.; Giugliano, R.P.; Keech, A.C.; Honarpour, N.; Wiviott, S.D.; Murphy, S.A.; Kuder, J.F.; Wang, H.; Liu, T.; Wasserman, S.M.; et al. Evolocumab and Clinical Outcomes in Patients with Cardiovascular Disease. N. Engl. J. Med. 2017, 376, 1713-1722. [CrossRef]

106. Schwartz, G.G.; Szarek, M.; Bhatt, D.L.; Bittner, V.; Diaz, R.; Edelberg, J.; Goodman, S.G.; Hanotin, C.; Harrington, R.; Jukema, J.W.; et al. Alirocumab in Patients after Acute Coronary Syndrome; American College of Cardiology: Orlando, FL, USA, 2018.

107. Kazi, D.S.; Moran, A.E.; Coxson, P.G.; Penko, J.; Ollendor, D.A.; Pearson, S.D.; Tice, J.A.; Guzman, D.; Bibbins-Domingo, K. Cost-effectiveness of PCSK9 Inhibitor Therapy in Patients With Heterozygous Familial Hypercholesterolemia or Atherosclerotic Cardiovascular Disease. JAMA 2016, 316, 743-753. [CrossRef]

108. Svatikova, A.; Kopecky, S.L. Cholesterol Management in the Era of PCSK9 Inhibitors. Curr. Cardiol. Rep. 2017, 19, 83. [CrossRef] 
109. Khatib, R.; McKee, M.; Shannon, H.; Chow, C.; Rangarajan, S.; Teo, K.; Wei, L.; Mony, P.; Mohan, V.; Gupta, R.; et al. Availability and affordability of cardiovascular disease medicines and their effect on use in high-income, middle-income, and low-income countries: An analysis of the PURE study data. Lancet 2016, 387, 61-69. [CrossRef]

110. Yusuf, S.; Islam, S.; Chow, C.K.; Rangarajan, S.; Dagenais, G.; Diaz, R.; Gupta, R.; Kelishadi, R.; Iqbal, R.; Avezum, A.; et al. Use of secondary prevention drugs for cardiovascular disease in the community in high-income, middle-income, and low-income countries (the PURE Study): A prospective epidemiological survey. Lancet 2011, 378, 1231-1243. [CrossRef]

111. Wirtz, V.J.; Kaplan, W.A.; Kwan, G.F.; Laing, R.O. Access to Medications for Cardiovascular Diseases in Lowand Middle-Income Countries. Circulation 2016, 133, 2076-2085. [CrossRef] [PubMed]

112. Beall, R.F.; Schwalm, J.-D.R.; Huffman, M.D.; McCready, T.; Yusuf, S.; Attaran, A. Could patents interfere with the development of a cardiovascular polypill? J. Trans. Med. 2016, 14, 242. [CrossRef] [PubMed]

113. Bangalore, S.; Kamalakkannan, G.; Parkar, S.; Messerli, F.H. Fixed-dose combinations improve medication compliance: A meta-analysis. Am. J. Med. 2007, 120, 713-719. [CrossRef] [PubMed]

114. Huffman, M.D. The Polypill: From Promise to Pragmatism. PLoS Med. 2015, 12, e1001862. [CrossRef] [PubMed]

115. Elley, C.R.; Gupta, A.K.; Webster, R.; Selak, V.; Jun, M.; Patel, A.; Rodgers, A.; Thom, S. The efficacy and tolerability of "polypills": Meta-analysis of randomised controlled trials. PLoS ONE 2012, 7, e52145. [CrossRef]

116. Gupta, A.K.; Arshad, S.; Poulter, N.R. Compliance, safety, and effectiveness of fixed-dose combinations of antihypertensive agents: A meta-analysis. Hypertens (Dallas Tex. 1979). 2010, 55, 399-407. [CrossRef]

117. Yusuf, S.; Pais, P.; Afzal, R.; Xavier, D.; Teo, K.; Eikelboom, J.; Sigamani, A.; Mohan, V.; Gupta, R.; Thomas, N.; et al. Effects of a polypill (Polycap) on risk factors in middle-aged individuals without cardiovascular disease (TIPS): A phase II, double-blind, randomised trial. Lancet 2009, 373, 1341-1351.

118. Rodgers, A.; Patel, A.; Berwanger, O.; Bots, M.; Grimm, R.; Grobbee, D.E.; Jackson, R.; Neal, B.; Neaton, J.; Poulter, N.; et al. An international randomised placebo-controlled trial of a four-component combination pill ("polypill") in people with raised cardiovascular risk. PLOS ONE 2011, 6, e19857.

119. Huffman, M.D.; Rao, K.D.; Pichon-Riviere, A.; Zhao, D.; Harikrishnan, S.; Ramaiya, K.; Ajay, V.S.; Goenka, S.; Calcagno, J.I.; Caporale, J.E.; et al. A cross-sectional study of the microeconomic impact of cardiovascular disease hospitalization in four low- and middle-income countries. PLoS ONE 2011, 6, e20821. [CrossRef] [PubMed]

120. Si, Y.; Zhou, Z.; Su, M.; Ma, M.; Xu, Y.; Heitner, J. Catastrophic healthcare expenditure and its inequality for households with hypertension: Evidence from the rural areas of Shaanxi Province in China. Int. J. Equity Health 2017, 16, 1-12. [CrossRef] [PubMed]

121. Sun, J.; Liabsuetrakul, T.; Fan, Y.; Mcneil, E. Protecting patients with cardiovascular diseases from catastrophic health expenditure and impoverishment by health finance reform. Trop. Med. Int. Health 2015, 20, 1846-1854. [CrossRef] [PubMed]

122. Baeza-Cruz, G.; Peniche-Otero, G.; Alva-Esqueda, M.E.; Naranjo-Muedano, M.; Soria-Suárez, N.; Morales-Flores, H.J. Análisis de costo de la enfermedad, del tratamiento, las complicaciones e intervenciones de la hipercolesterolemia en México en 2016. Value Health Reg. Issues 2018, 17, 56-63. [CrossRef] [PubMed]

123. Thow, A.M.; Downs, S.; Jan, S. A systematic review of the effectiveness of food taxes and subsidies to improve diets: Understanding the recent evidence. Nutr. Rev. 2014, 72, 551-565. [CrossRef]

124. Hawkes, C.; Organization, W.H. Nutrition Labels and Health Claims: The Global Regulatory Environment; World Health Organization: Geneva, Switzerland, 2004.

125. Colchero, M.A.; Popkin, B.M.; Rivera, J.A.; Ng, S.W. Beverage purchases from stores in Mexico under the excise tax on sugar sweetened beverages: Observational study. BMJ 2016, 352, h6704. [CrossRef]

126. Colchero, M.A.; Rivera-Dommarco, J.; Popkin, B.M.; Ng, S.W. In Mexico, Evidence Of Sustained Consumer Response Two Years After Implementing A Sugar-Sweetened Beverage Tax. Health Aff. 2017, 36, 564-571. [CrossRef]

127. Batis, C.; Rivera, J.A.; Popkin, B.M.; Taillie, L.S. First-Year Evaluation of Mexico's Tax on Nonessential Energy-Dense Foods: An Observational Study. PLoS Med. 2016, 13, e1002057. [CrossRef] 
128. Silver, L.D.; Ng, S.W.; Ryan-Ibarra, S.; Taillie, L.S.; Induni, M.; Miles, D.R.; Poti, J.M.; Popkin, B.M. Changes in prices, sales, consumer spending, and beverage consumption one year after a tax on sugar-sweetened beverages in Berkeley, California, US: A before-and-after study. PLoS Med. 2017, 14, e1002283. [CrossRef]

129. Falbe, J.; Thompson, H.R.; Becker, C.M.; Rojas, N.; McCulloch, C.E.; Madsen, K.A. Impact of the Berkeley Excise Tax on Sugar-Sweetened Beverage Consumption. Am. J. Public Health 2016, 106, 1865-1871. [CrossRef]

130. Bascunan, J.; Cuadrado, C. Effectiveness of sugar-sweetened beverages taxes to reduce obesity: Evidence brief for policy. Medwave 2017, 17, e7054. [CrossRef] [PubMed]

(C) 2019 by the authors. Licensee MDPI, Basel, Switzerland. This article is an open access article distributed under the terms and conditions of the Creative Commons Attribution (CC BY) license (http://creativecommons.org/licenses/by/4.0/). 